\title{
ON THE MODIFIED BOUNDARY VALUE PROBLEM OF DE LA VALLÉE-POUSSIN FOR NONLINEAR ORDINARY DIFFERENTIAL EQUATIONS
}

\author{
G. TSKHOVREBADZE
}

\begin{abstract}
The sufficient conditions of the existence, uniqueness, and correctness of the solution of the modified boundary value problem of de la Vallée-Poussin have been found for a nonlinear ordinary differential equation

$$
u^{(n)}=f\left(t, u, u^{\prime}, \ldots, u^{(n-1)}\right),
$$

where the function $f$ has nonitegrable singularities with respect to the first argument.
\end{abstract}

\section{$\S 1$. Statement of the Main Results}

In this paper for an ordinary differential equation

$$
u^{(n)}=f\left(t, u, u^{\prime}, \ldots, u^{(n-1)}\right)
$$

we shall consider the boundary value problem

$$
\begin{gathered}
u^{(k-1)}\left(t_{i}\right)=0 \quad\left(k=1, \ldots, n_{i} ; i=1, \ldots, m\right),{ }^{1} \\
\sup \left\{(t-a)^{l-1-\lambda_{1}}(b-t)^{l-1-\lambda_{2}}\left|u^{(l-1)}(t)\right|: a<t<b\right\}<+\infty,
\end{gathered}
$$

where $n \geq 2, l \in\{1, \ldots, n\}, m \in\{2, \ldots, n\},-\infty<a=t_{1}<\cdots<t_{m}=$ $\left.b<+\infty, n_{i} \in\{1, \ldots, n-1\}(i=1, \ldots, m), \sum_{i=1}^{m} n_{i}=n, \lambda_{1} \in\right] n_{1}-1, n_{1}[$, $\left.\lambda_{2} \in\right] n_{m}-1, n_{m}\left[, I_{m}=[a, b] \backslash\left\{t_{1}, \ldots, t_{m}\right\}\right.$ and the function $f: I_{m} \times \mathbb{R}^{n} \rightarrow$ $\mathbb{R}$ satisfies the Caratheodory conditions on each compactum contained in $I_{m} \times \mathbb{R}^{n}$.

Problem (1.1), (1.2 $)$ is the well-known boundary value problem of de la Vallée-Poussin and has been studied with sufficient thoroughness both when $f$ is integrable with respect to the first argument on $[a, b]$ (see, for example, [2] and references from [5]) and when $f$ has nonintegrable singularities at the

1991 Mathematics Subject Classification. 34B15.

${ }^{1}$ Here $u^{(i)}(a)\left(u^{(i)}(b)\right)$ denotes the right (left) limit of the function $u^{(i)}$ at the point $a(b)$. 
points $t_{1}, \ldots, t_{m}$ (see, for example, [5]-[7]). However, in the works devoted to the study of problem (1.1), (1.2 1$)$ it is assumed that

$$
\int_{a}^{b}(t-a)^{n-n_{1}-1}(b-t)^{n-n_{m}-1} f_{r}^{*}(t) d t<+\infty \text { for } r>0,
$$

where

$$
\begin{gathered}
f_{r}^{*}(t)=\max \left\{\left|f\left(t, \prod_{i=1}^{m}\left|t-t_{i}\right|^{n_{i 1}} x_{1}, \ldots, \prod_{i=1}^{m}\left|t-t_{i}\right|^{n_{i n}} x_{n}\right)\right|: \sum_{k=1}^{n}\left|x_{k}\right| \leq r\right\}, \\
n_{i k}= \begin{cases}n_{i}-k+1 & \text { for } k \leq n_{i}, \\
0 & \text { for } k>n_{i} .\end{cases}
\end{gathered}
$$

This assumption is not casual. The fact is that if condition (1.3) is not fulfilled, then problem (1.1), (1.21) is not, generally speaking, uniquely solvable even in the simplest case. For example, given the boundary condition $\left(1.2_{1}\right)$, the equation

$$
u^{(n)}=\frac{(-1)^{n} \delta}{(t-a)^{n}} u
$$

has an infinite number of solutions for $n_{1}=1$ and any sufficiently small $\delta>0$.

Therefore, for the solution to be unique, we have to introduce an additional and, of course, natural condition such as, for example, $\left(1.2_{2}\right)$. This condition is natural because if (1.3) is fulfilled, then (1.21) yields $\left(1.2_{2}\right)$, i.e., problem $(1.1),\left(1.2_{1}\right),\left(1.2_{2}\right)$ coincides with the problem of de la ValléePoussin (1.1), (1.2 1 ). However, if (1.3) is not fulfilled, then, as follows from the above example, this is not so.

Problem (1.1), (1.2 1$),\left(1.2_{2}\right)$ is the generalization of the boundary value problem of de la Vallée-Poussin $(1.1),\left(1.2_{1}\right)$ and has been studied in [14] ${ }^{2}$ for the linear differential equation

$$
u^{(n)}=\sum_{k=1}^{l} p_{k}(t) u^{(k-1)}+q(t),
$$

where $\left.p_{k}: I_{m} \rightarrow \mathbb{R}(k=1, \ldots, l), q:\right] a, b[\rightarrow \mathbb{R}$.

In this paper we shall establish the sufficient conditions for the existence, uniqueness, and correctness of the solution of problem (1.1), $\left(1.2_{1}\right),\left(1.2_{2}\right)$. Note that the solution of this problem is sought for in the class of functions $u:] a, b\left[\rightarrow \mathbb{R}\right.$ absolutely continuous with $u^{(k)}(k=1, \ldots, n-1)$ inside $] a, b\left[.^{3}\right.$

\footnotetext{
${ }^{2}$ See also [10]-[13].

${ }^{3}$ That is, on each segment contained in $] a, b[$.
} 
The following notation will be used:

$$
\begin{gathered}
\nu_{k l}=|(k-1-\lambda) \cdots(l-2-\lambda)| \quad(k=1, \ldots, l-1), \quad \nu_{l l}(\lambda)=1 ; \\
\sigma_{k, \lambda_{1}, \lambda_{2}}(t)=(t-a)^{\lambda_{1}-k+1}(b-t)^{\lambda_{2}-k+1} \prod_{i=2}^{m-1}\left|t-t_{i}\right|^{n_{i k}} ;{ }^{4} \\
\sigma_{k, n}(t)=(t-a)^{n-k}(b-t)^{n-k} \prod_{i=2}^{m-1}\left|t-t_{i}\right|^{n_{i k}} ; \\
\mathbb{R}=]-\infty,+\infty\left[; \quad \mathbb{R}_{+}=[0,+\infty[\right.
\end{gathered}
$$

$\mathbb{R}^{p}$, where $p$ is a natural number, is a $p$-dimensional Euclidean space;

$\mathbb{C}_{l o c}^{n-1}(] a, b[; \mathbb{R})$ is a set of functions $\left.v:\right] a, b[\rightarrow \mathbb{R}$ which are continuous, with $v^{(k)}(k=1, \ldots, n-1)$, inside $] a, b[$;

$\widetilde{\mathbb{C}}_{l o c}^{n-1}(] a, b[; \mathbb{R})$ is a set of functions $\left.v:\right] a, b[\rightarrow \mathbb{R}$ which are absolutely continuous, with $v^{(k)}(k=1, \ldots, n-1)$, inside $] a, b[$;

$L([a, b] ; I)$, where $I \subset \mathbb{R}$, is a set of functions $v:[a, b] \rightarrow I$ which are Lebesgue integrable on $[a, b]$;

$L_{l o c}(] a, b[; I)$ is a set of functions $\left.v:\right] a, b[\rightarrow I$ which are Lebesgue integrable inside $] a, b[$;

$L_{\alpha, \beta}(] a, b[; I)$, where $\alpha \geq 0, \beta \geq 0$, is a set of measurable functions $v:] a, b[\rightarrow I$ satisfying the condition

$$
|v(\cdot)|_{\alpha, \beta}=\sup \left\{(t-a)^{\alpha}(b-t)^{\beta}\left|\int_{\frac{a+b}{2}}^{t} v(\tau) d \tau\right|: a<t<b\right\}<+\infty ;
$$

$\mathbb{K}_{\text {loc }}\left(I \times \mathbb{R}^{p} ; \mathbb{R}\right)$, where $\left.I \in\right] a, b[$ is a measurable set and $p$ a natural number, is a set of functions $g: I \times \mathbb{R}^{p} \rightarrow \mathbb{R}$ satisfying the Caratheodory conditions on each compactum contained in $I \times \mathbb{R}^{p}$;

$\mathbb{K}_{\text {loc }}^{0}\left(I \times \mathbb{R}^{p} ; \mathbb{R}\right)$ is a set of functions $g: I \times \mathbb{R}^{p} \rightarrow \mathbb{R}$ such that $g\left(\cdot, x_{1}(\cdot), \ldots, x_{p}(\cdot)\right): I \rightarrow \mathbb{R}$ is a measurable function for any continuous vector function $\left(x_{1}, \ldots, x_{p}\right): I \rightarrow \mathbb{R}^{p}$

$\mathcal{D}_{\lambda_{1}, \lambda_{2}}\left(u_{0} ; r\right)$, where $r \in \mathbb{R}_{+}, u_{0} \in \mathbb{C}_{\text {loc }}^{n-1}(] a, b[; \mathbb{R})$ is a set of vectors $\left(x_{1}, \ldots, x_{l}\right) \in \mathbb{R}^{l}$ satisfying the condition

$$
\inf \left\{\sum_{k=1}^{l}\left|x_{k}-\frac{u_{0}^{(k-1)}(t)}{\sigma_{k, \lambda_{1}, \lambda_{2}}(t)}\right|: t \in I_{m}\right\} \leq r
$$

\footnotetext{
${ }^{4} \prod_{i=2}^{m-1}\left|t-t_{i}\right|^{n_{i k}}$ will denote unity when $m=2$.
} 
$W_{\lambda_{1}, \lambda_{2}}\left(u_{0} ; r\right)$ is a set of functions $u \in \mathbb{C}_{l o c}^{n-1}(] a, b[; \mathbb{R})$ satisfying the condition

$$
\sum_{k=1}^{l} \frac{\left|u^{(k-1)}(t)-u_{0}^{(k-1)}(t)\right|}{\sigma_{k, \lambda_{1}, \lambda_{2}}(t)} \leq r \quad \text { for } \quad a<t<b ;
$$

$M\left([\alpha, \beta] \times \mathbb{R}_{+} ; \mathbb{R}_{+}\right)$, where $a \leq \alpha<\beta \leq b$, is a set of functions $\omega \in$ $\mathbb{K}_{\text {loc }}\left([\alpha, \beta] \times \mathbb{R}_{+} ; \mathbb{R}_{+}\right)$which are nondecreasing with respect to the second argument and satisfy the condition $\omega(t, 0)=0$ for $\alpha \leq t \leq \beta$.

Throughout the paper it will be assumed that $f \in \mathbb{K}_{l o c}\left(I_{m} \times \mathbb{R}^{n} ; \mathbb{R}\right)$ and the solution of problem (1.1), (1.21), (1.22) will be sought for in the class $\widetilde{\mathbb{C}}_{l o c}^{n-1}(] a, b[; \mathbb{R})$.

Some definitions will be given.

Definition 1.1. Let $n_{0} \in\{1, \ldots, n-1\}$ and $\left.\lambda \in\right] n_{0}-1, n_{0}[$. A vector function $\left(h_{1}, \ldots, h_{l}\right)$ with measurable components $\left.h_{k}:\right] a, b[\rightarrow \mathbb{R}(k=$ $1, \ldots, l)$ is said to belong to the set $S^{+}\left(a, b ; n, n_{0} ; \lambda\right)\left(S^{-}\left(a, b ; n, n_{0} ; \lambda\right)\right)$ if there exists $\alpha \in] a, b[$ such that we have the inequality

$$
\begin{gathered}
\limsup _{t \rightarrow a} \frac{(t-a)^{l-1-\lambda}}{(n-l) !} \sum_{k=1}^{l} \frac{1}{\nu_{k l}(\lambda)} \int_{t}^{\alpha}(\tau-t)^{n-l}(\tau-a)^{\lambda-k+1}\left|h_{k}(\tau)\right| d \tau<1 \\
{\left[\limsup _{t \rightarrow b} \frac{(b-t)^{l-1-\lambda}}{(n-l) !} \sum_{k=1}^{l} \frac{1}{\nu_{k l}(\lambda)} \int_{\alpha}^{t}(t-\tau)^{n-l}(b-\tau)^{\lambda-k+1}\left|h_{k}(\tau)\right| d \tau<1\right]}
\end{gathered}
$$

in the case $l \in\left\{n_{0}+1, \ldots, n\right\}$ and the inequality

$$
\begin{gathered}
\limsup _{t \rightarrow a} \frac{(t-a)^{l-1-\lambda}}{\left(n-n_{0}-1\right) !\left(n_{0}-l\right) !} \times \\
\times \sum_{k=1}^{l} \frac{1}{\nu_{k l}(\lambda)} \int_{a}^{t}(t-s)^{n_{0}-l} \int_{s}^{\alpha}(\tau-s)^{n-n_{0}-1}(\tau-a)^{\lambda-k+1}\left|h_{k}(\tau)\right| d \tau d s<1 \\
{\left[\limsup _{t \rightarrow b} \frac{(b-t)^{l-1-\lambda}}{\left(n-n_{0}-1\right) !\left(n_{0}-l\right) !} \times\right.} \\
\left.\times \sum_{k=1}^{l} \frac{1}{\nu_{k l}(\lambda)} \int_{t}^{b}(s-t)^{n_{0}-l} \int_{\alpha}^{s}(s-\tau)^{n-n_{0}-1}(b-\tau)^{\lambda-k+1}\left|h_{k}(\tau)\right| d \tau d s<1\right]
\end{gathered}
$$

in the case $l \in\left\{1, \ldots, n_{0}\right\}$. 


\section{Definition 1.2. Let}

$$
\begin{gathered}
\sigma_{k, \lambda_{1}, \lambda_{2}}(\cdot) p_{j k}(\cdot) \in L_{l o c}(] a, b[; \mathbb{R}) \quad(j=1,2 ; k=1, \ldots, l), \\
p_{1 k}(t) \leq p_{2 k}(t) \quad \text { for } \quad a<t<b(k=1, \ldots, l), \\
\left(p_{1}^{*}, \ldots, p_{l}^{*}\right) \in S^{+}\left(a, b ; n, n_{1} ; \lambda_{1}\right) \cap S^{-}\left(a, b ; n, n_{m} ; \lambda_{2}\right),
\end{gathered}
$$

where $p_{k}^{*}(t)=\max \left\{\left|p_{1 k}(t)\right|,\left|p_{2 k}(t)\right|\right\}(k=1, \ldots, l)$ and, moreover, under the boundary conditions $\left(1.2_{1}\right),\left(1.2_{2}\right)$ the differential equation

$$
u^{(n)}=\sum_{k=1}^{l} p_{k}(t) u^{(k-1)}
$$

haa no nontrivial solution no matter what the measurable functions $p_{k}$ : ]$a, b[\rightarrow \mathbb{R}(k=1, \ldots, l)$ satisfying the inequalities

$$
p_{1 k}(t) \leq p_{k}(t) \leq p_{2 k}(t) \text { for } a<t<b \quad(k=1, \ldots, l)
$$

are. Then the vector function $\left(p_{11}, \ldots, p_{1 l} ; p_{21}, \ldots, p_{2 l}\right)$ is said to belong to the class $V\left(t_{1}, \ldots, t_{m} ; n_{1}, \ldots, n_{m} ; \lambda_{1}, \lambda_{2}\right)$.

\subsection{EXISTEnCE AND Uniqueness Theorems}

\section{The General Case.}

Theorem 1.1. Let the following inequalities be fulfilled on $] a, b\left[\times \mathbb{R}^{n}\right.$ :

$$
\begin{gathered}
\left|f\left(t, x_{1}, \ldots, x_{n}\right)-\sum_{k=1}^{l} p_{k}\left(t, x_{1}, \ldots, x_{n}\right) x_{k}-q_{0}(t)\right| \leq \\
\leq q\left(t, \sum_{k=1}^{l} \frac{\left|x_{k}\right|}{\sigma_{k, \lambda_{1}, \lambda_{2}}(t)}\right)
\end{gathered}
$$

and

$$
p_{1 k}(t) \leq p_{k}\left(t, x_{1}, \ldots, x_{n}\right) \leq p_{2 k}(t) \quad(k=1, \ldots, l)
$$

where

$$
\left(p_{11}, \ldots, p_{1 l} ; p_{21}, \ldots, p_{2 l}\right) \in V\left(t_{1}, \ldots, t_{m} ; n_{1}, \ldots, n_{m} ; \lambda_{1}, \lambda_{2}\right),
$$

$p_{k} \in \mathbb{K}_{\text {loc }}^{0}\left(I_{m} \times \mathbb{R}^{n} ; \mathbb{R}\right) \quad(k=1, \ldots, l), \quad q_{0} \in L_{n-1-\lambda_{1}, n-1-\lambda_{2}}(] a, b[; \mathbb{R})$,

the function $q:] a, b\left[\times \mathbb{R}_{+} \rightarrow \mathbb{R}_{+}\right.$is nondecreasing with respect to the second argument, $q(\cdot, \varrho) \in L_{n-1-\lambda_{1}, n-1-\lambda_{2}}(] a, b\left[; \mathbb{R}_{+}\right)$for any $\varrho \geq 0$, and

$$
\lim _{\varrho \rightarrow+\infty} \frac{|q(\cdot, \varrho)|_{n-1-\lambda_{1}, n_{1}-1-\lambda_{2}}}{\varrho}=0 .
$$

Then problem (1.1), $\left(1.2_{1}\right),\left(1.2_{2}\right)$ is solvable. 
Corollary 1.1. Let the following inequality be fulfilled:

$$
\begin{gathered}
\left|f\left(t, x_{1}, \ldots, x_{n}\right)-q_{0}(t)\right| \leq \sum_{k=1}^{l} p_{k}(t)\left|x_{k}\right|+ \\
+q\left(t, \sum_{k=1}^{l} \frac{\left|x_{k}\right|}{\sigma_{k, \lambda_{1}, \lambda_{2}}(t)}\right)
\end{gathered}
$$

where

$$
\begin{gathered}
q_{0} \in L_{n-1-\lambda_{1}, n-1-\lambda_{2}}(] a, b[; \mathbb{R}), \\
\left(-p_{1}, \ldots,-p_{l} ; p_{1}, \ldots, p_{l}\right) \in V\left(t_{1}, \ldots, t_{m} ; n_{1}, \ldots, n_{m} ; \lambda_{1}, \lambda_{2}\right),
\end{gathered}
$$

and the function $q:] a, b\left[\times \mathbb{R}_{+} \rightarrow \mathbb{R}_{+}\right.$satisfy the conditions of Theorem 1.1. Then problem $(1.1),\left(1.2_{1}\right),\left(1.2_{2}\right)$ is solvable.

For the case where the right-hand side of equation (1.1) is independent of the last $n-l$ arguments, i.e.,

$$
u^{(n)}=f\left(t, u, \ldots, u^{(l-1)}\right)
$$

we have

Theorem 1.2. Let $f(\cdot, 0, \ldots, 0) \in L_{n-1-\lambda_{1}, n-1-\lambda_{2}}(] a, b[; \mathbb{R})$ and the function $f$ have partial derivatives with respect to phase arguments belonging to $\mathbb{K}_{\text {loc }}\left(I_{m} \times \mathbb{R}^{l} ; \mathbb{R}\right)$. Let, moreover, the following inequalities be fulfilled on $] a, b\left[\times \mathbb{R}^{l}\right.$ :

$$
p_{1 k}(t) \leq \frac{\partial f\left(t, x_{1}, \ldots, x_{l}\right)}{\partial x_{k}} \leq p_{2 k}(t) \quad(k=1, \ldots, l),
$$

and $\left(p_{11}, \ldots, p_{1 l} ; p_{21}, \ldots, p_{2 l}\right) \in V\left(t_{1}, \ldots, t_{m} ; n_{1}, \ldots, n_{m} ; \lambda_{1}, \lambda_{2}\right)$. Then problem $\left(1.1^{\prime}\right),\left(1.2_{1}\right),\left(1.2_{2}\right)$ has unique solution.

The case $l=1$.

In this subsection we shall consider the problem

$$
\begin{gathered}
u^{(n)}=f(t, u), \\
u^{(k-1)}\left(t_{i}\right)=0 \quad\left(k=1, \ldots, n_{i} ; i=1, \ldots, m\right), \\
\sup \left\{(t-a)^{-\lambda_{1}}(b-t)^{-\lambda_{2}}|u(t)|: a<t<b\right\}<+\infty,
\end{gathered}
$$

assuming that $f \in \mathbb{K}_{l o c}\left(I_{m} \times \mathbb{R} ; \mathbb{R}\right)$.

For any $r \in L_{n-1-\lambda_{1}, n-1-\lambda_{2}}(] a, b[; \mathbb{R})$ we introduce the notation

$$
\varrho_{\lambda_{1}, \lambda_{2}}(r)=\text { vraimax }\left\{\frac{\left|u_{0}(r)(t)\right|}{\sigma_{1, \lambda_{1}, \lambda_{2}}(t)}: a<t<b\right\}
$$


where $u_{0}(r)(\cdot)$ is the unique solution of the equation $u^{(n)}=r(t)$ satisfying the boundary conditions $\left(1.14_{1}\right),\left(1.14_{2}\right)$ (see, for example, Proposition 2.3).

Theorem 1.3. Let the following inequality be fulfilled on $] a, b[\times \mathbb{R}$ :

$$
\left|f(t, x)-q_{0}(t)\right| \leq \frac{r(t)}{\sigma_{1, \lambda_{1}, \lambda_{2}}(t)}|x|+q\left(t, \frac{|x|}{\sigma_{1, \lambda_{1}, \lambda_{2}}(t)}\right),
$$

where

$$
q_{0}, r \in L_{n-1-\lambda_{1}, n-1-\lambda_{2}}(] a, b[; \mathbb{R}),
$$

and let the function $q:] a, b\left[\times \mathbb{R}_{+} \rightarrow \mathbb{R}_{+}\right.$be nondecreasing with respect to the second argument, $q(\cdot, \varrho) \in L_{n-1-\lambda_{1}, n-1-\lambda_{2}}(] a, b\left[; \mathbb{R}_{+}\right)$for any $\varrho \geq 0$ and

$$
\lim _{\varrho \rightarrow+\infty} \frac{|q(\cdot, \varrho)|_{n-1-\lambda_{1}, n-1-\lambda_{2}}}{\varrho}=0 .
$$

Let, moreover,

$$
\varrho_{\lambda_{1}, \lambda_{2}}(r)<1 .
$$

Then problem (1.13), (1.14 $),\left(1.14_{2}\right)$ is solvable.

Corollary 1.2. Let $m=2$ and the following inequality be fulfilled on ]$a, b[\times \mathbb{R}$ :

$$
\left|f(t, x)-q_{0}(t)\right| \leq \frac{r_{0}}{(t-a)^{n}(b-t)^{n}}|x|+q\left(t, \frac{|x|}{(t-a)^{\lambda_{1}}(b-t)^{\lambda_{2}}}\right),
$$

where the functions $q_{0}$ and $q$ satisfy the conditions of Theorem 1.3, and the number $r_{0}$ the inequality

$$
\begin{aligned}
& r_{0}<\left[n_{1} ! n_{2} !\left(\lambda_{1}-n_{1}+1\right)\left(\lambda_{2}-n_{2}+1\right)\left(n_{1}-\lambda_{1}\right)\left(n_{2}-\lambda_{2}\right)(b-a)^{n}\right] \times \\
& \quad \times\left[2 n_{1}\left(\lambda_{2}-n_{2}+1\right)\left(n_{1}-\lambda_{1}\right)\left(\lambda_{1}-n_{1}+1+n_{2}-\lambda_{2}\right)+\right. \\
& \left.+2 n_{2}\left(\lambda_{1}-n_{1}+1\right)\left(n_{2}-\lambda_{2}\right)\left(\lambda_{2}-n_{2}+1+n_{1}-\lambda_{1}\right)\right]^{-1} .
\end{aligned}
$$

Then problem $(1.13),\left(1.14_{1}\right),\left(1.14_{2}\right)$ is solvable.

For the two-point boundary value problem

$$
\begin{gathered}
u^{\prime \prime}=f(t, u), \\
u(a)=u(b)=0, \\
\sup \left\{(t-a)^{-\lambda_{1}}(b-t)^{-\lambda_{2}}|u(t)|: a<t<b\right\}<+\infty,
\end{gathered}
$$

where $0<\lambda_{1}, \lambda_{2}<1$, and for the three-point boundary value problem

$$
\begin{gathered}
u^{\prime \prime \prime}=f(t, u), \\
u(a)=u\left(t_{0}\right)=u(b)=0, \\
\sup \left\{(t-a)^{-\lambda_{1}}(b-t)^{-\lambda_{2}}|u(t)|: a<t<b\right\}<+\infty,
\end{gathered}
$$


where $a<t_{0}<b, 0<\lambda_{1}, \lambda_{2}<1$, from Theorem 1.3 we obtain

Corollary 1.3. Let the following inequality be fulfilled on $] a, b[\times \mathbb{R}$ :

$$
\begin{aligned}
\left|f(t, x)-q_{0}(t)\right| \leq r_{0}( & \left.\frac{\lambda_{1}\left(1-\lambda_{1}\right)}{(t-a)^{2}}+\frac{2 \lambda_{1} \lambda_{2}}{(t-a)(b-t)}+\frac{\lambda_{2}\left(1-\lambda_{2}\right)}{(b-t)^{2}}\right)|x|+ \\
& +q\left(t, \frac{|x|}{(t-a)^{\lambda_{1}}(b-t)^{\lambda_{2}}}\right),
\end{aligned}
$$

where $\left.r_{0} \in\right] 0,1\left[\right.$ and the functions $q_{0}$ and $q$ satisfy the conditions of Theorem 1.3 for $n=m=2, n_{1}=n_{2}=1$. Then problem (1.18), (1.191 $),\left(1.19_{2}\right)$ is solvable.

Corollary 1.4. Let the following inequality be fulfilled on $] a, b[\times \mathbb{R}$ :

$$
\begin{gathered}
\left|f(t, x)-q_{0}(t)\right| \leq r_{0} \mid \frac{\lambda_{1}\left(1-\lambda_{1}\right)\left(2-\lambda_{1}\right)}{(t-a)^{3}}-\frac{3 \lambda_{1}\left(1-\lambda_{1}\right)}{(t-a)^{2}\left(t-t_{0}\right)}+ \\
+\frac{3 \lambda_{1} \lambda_{2}\left(1-\lambda_{1}\right)}{(t-a)^{2}(b-t)}-\frac{6 \lambda_{1} \lambda_{2}}{(t-a)\left(t-t_{0}\right)(b-t)}-\frac{3 \lambda_{1} \lambda_{2}\left(1-\lambda_{2}\right)}{(t-a)(b-t)^{2}}- \\
-\frac{3 \lambda_{2}\left(1-\lambda_{2}\right)}{\left(t-t_{0}\right)(b-t)^{2}}-\frac{\lambda_{2}\left(1-\lambda_{2}\right)\left(2-\lambda_{2}\right)}{(b-t)^{3}}|| x \mid+ \\
+q\left(t, \frac{|x|}{(t-a)^{\lambda_{1}}\left|t-t_{0}\right|(b-t)^{\lambda_{2}}}\right),
\end{gathered}
$$

where $\left.r_{0} \in\right] 0,1\left[\right.$ and the functions $q_{0}$ and $q$ satisfy the conditions of Theorem 1.3 for $n=m=3, n_{1}=n_{2}=n_{3}=1$. Then problem (1.20), (1.21 $),\left(1.21_{2}\right)$ is solvable.

Theorem 1.4. Let $f(\cdot, 0) \in L_{n-1-\lambda_{1}, n-1-\lambda_{2}}(] a, b[; \mathbb{R})$ and the following inequality be fulfilled on $] a, b[\times \mathbb{R}$ :

$$
|f(t, x)-f(t, y)| \leq \frac{r(t)}{\sigma_{1, \lambda_{1}, \lambda_{2}}(t)}|x-y|
$$

where the function $r \in L_{n-1-\lambda_{1}, n-1-\lambda_{2}}(] a, b[; \mathbb{R})$ satisfies condition (1.16). Then problem (1.13), $\left(1.14_{1}\right),\left(1.14_{2}\right)$ has the unique solution $u$ and

$$
\text { vrai max }\left\{\frac{\left|u_{j}(t)-u(t)\right|}{\sigma_{1, \lambda_{1}, \lambda_{2}}(t)}: a<t<b\right\} \rightarrow 0 \text { for } j \rightarrow+\infty,
$$

where $u_{0}(t) \equiv 0$, and for each natural number $j$ the function $u_{j}$ is a solution of the equation

$$
u_{j}^{(n)}(t)=f\left(t, u_{j-1}(t)\right),
$$

satisfying the boundary conditions $\left(1.14_{1}\right),\left(1.14_{2}\right)$. 
Corollary 1.5. Let $m=2, f(\cdot, 0) \in L_{n-1-\lambda_{1}, n-1-\lambda_{2}}(] a, b[; \mathbb{R})$ and the following inequality be fulfilled on $] a, b[\times \mathbb{R}$ :

$$
|f(t, x)-f(t, y)| \leq \frac{r_{0}}{(t-a)^{n}(b-t)^{n}}|x-y|
$$

where $r_{0}$ is a number satisfying (1.17). Then problem $(1.13),\left(1.14_{1}\right),\left(1.14_{2}\right)$ has the unique solution $u$ and

$$
\text { vrai } \max \left\{\frac{\left|u_{j}(t)-u(t)\right|}{(t-a)^{\lambda_{1}}(b-t)^{\lambda_{2}}}: a<t<b\right\} \rightarrow 0 \text { for } j \rightarrow+\infty,
$$

where $u_{0}(t) \equiv 0$, and for each natural number $j$ the function $u_{j}$ is a solution of problem (1.24), (1.14 $),\left(1.14_{2}\right)$.

Corollary 1.6. Let $f(\cdot, 0) \in L_{1-\lambda_{1}, 1-\lambda_{2}}(] a, b[; \mathbb{R})$ and the following inequality be fulfilled on $] a, b[\times \mathbb{R}$ :

$$
|f(t, x)-f(t, y)| \leq r_{0}\left(\frac{\lambda_{1}\left(1-\lambda_{1}\right)}{(t-a)^{2}}+\frac{2 \lambda_{1} \lambda_{2}}{(t-a)(b-t)}+\frac{\lambda_{2}\left(1-\lambda_{2}\right)}{(b-t)^{2}}\right)|x-y|,
$$

where $\left.r_{0} \in\right] 0,1\left[\right.$. Then problem (1.18), $\left(1.19_{1}\right),\left(1.19_{2}\right)$ has the unique solution $u$ and

$$
\text { vraimax }\left\{\frac{\left|u_{j}(t)-u(t)\right|}{(t-a)^{\lambda_{1}}(b-t)^{\lambda_{2}}}: a<t<b\right\} \rightarrow 0 \text { for } j \rightarrow+\infty,
$$

where $u_{0}(t) \equiv 0$, and for each natural number $j$ the function $u_{j}$ is a solution of the equation $u_{j}^{\prime \prime}(t)=f\left(t, u_{j-1}(t)\right)$, satisfying the boundary conditions $\left(1.19_{1}\right),\left(1.19_{2}\right)$.

Corollary 1.7. Let $f(\cdot, 0) \in L_{2-\lambda_{1}, 2-\lambda_{2}}(] a, b[; \mathbb{R})$ and the following inequality be fulfilled on $] a, b[\times \mathbb{R}$ :

$$
\begin{gathered}
|f(t, x)-f(t, y)| \leq r_{0} \mid \frac{\lambda_{1}\left(1-\lambda_{1}\right)\left(2-\lambda_{1}\right)}{(t-a)^{3}}-\frac{3 \lambda_{1}\left(1-\lambda_{1}\right)}{(t-a)^{2}\left(t-t_{0}\right)}+ \\
+\frac{3 \lambda_{1} \lambda_{2}\left(1-\lambda_{1}\right)}{(t-a)^{2}(b-t)}-\frac{6 \lambda_{1} \lambda_{2}}{(t-a)\left(t-t_{0}\right)(b-t)}-\frac{3 \lambda_{1} \lambda_{2}\left(1-\lambda_{2}\right)}{(t-a)(b-t)^{2}}- \\
\quad-\frac{3 \lambda_{2}\left(1-\lambda_{2}\right)}{\left(t-t_{0}\right)(b-t)^{2}}-\frac{\lambda_{2}\left(1-\lambda_{2}\right)\left(2-\lambda_{2}\right)}{(b-t)^{3}}|| x-y \mid,
\end{gathered}
$$

where $\left.r_{0} \in\right] 0,1\left[\right.$. Then problem (1.20), $\left(1.21_{1}\right),\left(1.21_{2}\right)$ has the unique solution $u$ and

$$
\text { vrai } \max \left\{\frac{\left|u_{j}(t)-u(t)\right|}{(t-a)^{\lambda_{1}}\left|t-t_{0}\right|(b-t)^{\lambda_{2}}}: a<t<b\right\} \rightarrow 0 \text { for } j \rightarrow+\infty,
$$

where $u_{0}(t) \equiv 0$, and for each natural number $j$ the function $u_{j}$ is a solution of the equation $u_{j}^{\prime \prime \prime}(t)=f\left(t, u_{j-1}(t)\right)$, satisfying the boundary conditions $\left(1.21_{1}\right),\left(1.21_{2}\right)$. 
Remark 1.1. In Corollaries 1.3, 1.4, 1.6, and 1.7 the condition

$$
\left.r_{0} \in\right] 0,1[
$$

cannot be replaced by the equality

$$
r_{0}=1 \text {. }
$$

\subsection{Continuous Dependence of Solutions of the Right-Hand Side of the Equation}

In this subsection we shall consider the boundary value problem

$$
\begin{gathered}
u^{(n)}=f\left(t, u, u^{\prime}, \ldots, u^{(l-1)}\right), \\
u^{(k-1)}\left(t_{i}\right)=0 \quad\left(k=1, \ldots, n_{i} ; i=1, \ldots, m\right), \\
\sup \left\{(t-a)^{l-1-\lambda_{1}}(b-t)^{l-1-\lambda_{2}}\left|u^{(l-1)}(t)\right|: a<t<b\right\}<+\infty,
\end{gathered}
$$

assuming that $f, \frac{\partial f}{\partial x_{k}} \in \mathbb{K}_{l o c}\left(I_{m} \times \mathbb{R}^{l} ; \mathbb{R}\right)(k=1, \ldots, l)$, and give the sufficient conditions for its solutions to be stable with respect to small perturbations of the right-hand side of equation $\left(1.1^{\prime}\right)$.

Definition 1.3. Let $u_{0}$ be a solution of problem $\left(1.1^{\prime}\right),\left(1.2_{1}\right),\left(1.2_{2}\right)$ and $r$ be a positive number. It will be said that $u_{0}$ is $r$-stable with respect to small perturbations of the right-hand side of equation $\left(1.1^{\prime}\right)$ if for any $\varepsilon \in] 0, r[, \alpha \in] a, t_{2}[, \beta \in] t_{m-1}, b\left[,\left(x_{10}, \ldots, x_{l 0}\right) \in \mathcal{D}_{\lambda_{1}, \lambda_{2}}\left(u_{0} ; r\right)\right.$ and $\omega \in M\left([\alpha, \beta] \times \mathbb{R}_{+} ; \mathbb{R}_{+}\right)$there exists $\delta>0$ such that for any function $\eta \in \mathbb{K}_{\text {loc }}\left(I_{m} \times \mathbb{R}^{l} ; \mathbb{R}\right)$ satisfying the conditions

$$
\begin{gathered}
\mid \begin{array}{c}
\left|\int_{\alpha}^{t} \eta\left(\tau, \sigma_{1, \lambda_{1}, \lambda_{2}}(\tau) x_{1}, \ldots, \sigma_{l, \lambda_{1}, \lambda_{2}}(\tau) x_{l}\right) d \tau\right| \leq \delta \\
\text { for } \alpha \leq t \leq \beta,\left(x_{1}, \ldots, x_{l}\right) \in \mathcal{D}_{\lambda_{1}, \lambda_{2}}\left(u_{0} ; r\right), \\
\mid \eta\left(t, \sigma_{1, \lambda_{1}, \lambda_{2}}(t) x_{1}, \ldots, \sigma_{l, \lambda_{1}, \lambda_{2}}(t) x_{l}\right)- \\
-\eta\left(t, \sigma_{1, \lambda_{1}, \lambda_{2}}(t) y_{1}, \ldots, \sigma_{l, \lambda_{1}, \lambda_{2}}(t) y_{l}\right) \mid \leq \omega\left(t, \sum_{k=1}^{l}\left|x_{k}-y_{k}\right|\right) \\
\text { for } \alpha \leq t \leq \beta, \quad\left(x_{1}, \ldots, x_{l}\right) \text { and }\left(y_{1}, \ldots, y_{l}\right) \in \mathcal{D}_{\lambda_{1}, \lambda_{2}}\left(u_{0} ; r\right), \\
(t-a)^{n-1-\lambda_{1}}\left[\int_{t}^{\alpha} \eta_{\lambda_{1}, \lambda_{2}}^{*}\left(\tau ; u_{0} ; r\right) d \tau+\left|\int_{t}^{\alpha} \eta_{0}(\tau) d \tau\right|\right] \leq \delta \\
\text { for } a<t \leq \alpha,
\end{array}
\end{gathered}
$$




$$
\begin{gathered}
(b-t)^{n-1-\lambda_{2}}\left[\int_{\beta}^{t} \eta_{\lambda_{1}, \lambda_{2}}^{*}\left(\tau ; u_{0} ; r\right) d \tau+\left|\int_{\beta}^{t} \eta_{0}(\tau) d \tau\right|\right] \leq \delta \\
\text { for } \beta \leq t<b,
\end{gathered}
$$

where

$$
\begin{gathered}
\eta_{0}(t)=\eta\left(t, \sigma_{1, \lambda_{1}, \lambda_{2}}(t) x_{10}, \ldots, \sigma_{l, \lambda_{1}, \lambda_{2}}(t) x_{l 0}\right), \\
\eta_{\lambda_{1}, \lambda_{2}}^{*}\left(t ; u_{0} ; r\right)=\sup \left\{\left|\eta\left(t, \sigma_{1, \lambda_{1}, \lambda_{2}}(t) x_{1}, \ldots, \sigma_{l, \lambda_{1}, \lambda_{2}}(t) x_{l}\right)-\eta_{0}(t)\right|:\right. \\
\left.\left(x_{1}, \ldots, x_{l}\right) \mathcal{D}_{\lambda_{1}, \lambda_{2}}\left(u_{0}, r\right)\right\},
\end{gathered}
$$

the equation $u^{(n)}=f\left(t, u, \ldots, u^{(l-1)}\right)+\eta\left(t, u, \ldots, u^{(l-1)}\right)$ has at least one solution in $W_{\lambda_{1}, \lambda_{2}}\left(u_{0} ; r\right)$, and every such solution is also contained in $W_{\lambda_{1}, \lambda_{2}}\left(u_{0} ; \varepsilon\right)$.

Definition 1.4. It will be said that the solution $u_{0}$ of problem $\left(1.1^{\prime}\right)$, $\left(1.2_{1}\right),\left(1.2_{2}\right)$ is stable with respect to small perturbations of the right-hand side of equation $\left(1.1^{\prime}\right)$ if it is $r$-stable for any $r>0$.

Theorem 1.5. Let $u_{0}$ be a solution of problem $\left(1.1^{\prime}\right),\left(1.2_{1}\right),\left(1.2_{2}\right), r>$ 0 , and let the following inequalities be fulfilled on $] a, b\left[\times \mathcal{D}_{\lambda_{1}, \lambda_{2}}\left(u_{0} ; r\right)\right.$ :

$$
\begin{gathered}
p_{1 k}(t) \leq \frac{\partial f\left(t, \sigma_{1, \lambda_{1}, \lambda_{2}}(t) x_{1}, \ldots, \sigma_{l, \lambda_{1}, \lambda_{2}}(t) x_{l}\right)}{\partial x_{k} \quad} \leq p_{2 k}(t) \\
(k=1, \ldots, l),
\end{gathered}
$$

where

$$
\left(p_{11}, \ldots, p_{1 l} ; p_{21}, \ldots, p_{2 l}\right) \in V\left(t_{1}, \ldots, t_{m} ; n_{1}, \ldots, n_{m} ; \lambda_{1}, \lambda_{2}\right) .
$$

Then $u_{0}$ is $r$-stable with respect to small perturbations of the right-hand side of equation $\left(1.1^{\prime}\right)$.

Theorem 1.6. Let

$$
f(\cdot, 0, \ldots, 0) \in L_{n-1-\lambda_{1}, n-1-\lambda_{2}}(] a, b[; \mathbb{R})
$$

and the following inequalities be fulfilled on $] a, b\left[\times \mathbb{R}^{l}\right.$ :

$$
p_{1 k}(t) \leq \frac{\partial f\left(t, x_{1}, \ldots, x_{l}\right)}{\partial x_{k}} \leq p_{2 k}(t) \quad(k=1, \ldots, l),
$$

where $p_{j k}(j=1,2 ; k=1, \ldots, l)$ satisfy condition (1.8). Then problem $\left(1.1^{\prime}\right),\left(1.2_{1}\right),\left(1.2_{2}\right)$ has the unique solution $u_{0}$ and, moreover, this solution is stable with respect to small perturbations of the right-hand side of equation $\left(1.1^{\prime}\right)$. 


\section{$\S 2$. Auxiliary Propositions}

Proposition 2.1. Let

$$
\left(p_{11}, \ldots, p_{1 l} ; p_{21}, \ldots, p_{2 l}\right) \in V\left(t_{1}, \ldots, t_{m} ; n_{1}, \ldots, n_{m} ; \lambda_{1}, \lambda_{2}\right) .
$$

Then there exists a positive number $\varrho_{0}$ such that for any $q \in L_{n-1-\lambda_{1}, n-1-\lambda_{2}}(] a, b[; \mathbb{R})$ and measurable functions $\left.p_{k}:\right] a, b[\rightarrow \mathbb{R}(k=$ $1, \ldots, l)$ satisfying inequalities (1.5) an arbitrary solution u of problem (1.4), $\left(1.2_{1}\right),\left(1.2_{2}\right)$ admits the estimate

$$
\begin{gathered}
\left|u^{(k-1)}(t)\right| \leq \varrho_{0} \sigma_{k, \lambda_{1}, \lambda_{2}}(t)|q(\cdot)|_{n-1-\lambda_{1}, n-1-\lambda_{2}} \\
\text { for } a<t<b \quad(k=1, \ldots, l) .
\end{gathered}
$$

Proposition 2.2. Let

$$
\sigma_{k, \lambda_{1}, \lambda_{2}}(\cdot) p_{k}(\cdot) \in L_{l o c}(] a, b[; \mathbb{R}) \quad(k=1, \ldots, l)
$$

and

$$
\left(p_{1}, \ldots, p_{l}\right) \in S^{+}\left(a, b ; n, n_{1} ; \lambda_{1}\right) \cap S^{-}\left(a, b ; n, n_{m} ; \lambda_{2}\right) .
$$

Then for problem (1.4), (1.2 $),\left(1.2_{2}\right)$ to be uniquely solvable for each $q \in$ $L_{n-1-\lambda_{1}, n-1-\lambda_{2}}(] a, b[; \mathbb{R})$ it is necessary and sufficient that the corresponding homogeneous problem $\left(1.4_{0}\right),\left(1.2_{1}\right),\left(1.2_{2}\right)$ have the trivial solution only.

Proposition 2.3. Let

$$
p_{k}(t)=\frac{g_{1 k}(t)}{(t-a)^{n-k+1}}+\frac{g_{2 k}(t)}{(b-t)^{n-k+1}}+p_{0 k}(t) \quad(k=1, \ldots, l),
$$

where $\sigma_{k, n}(\cdot) p_{0 k}(\cdot) \in L([a, b] ; \mathbb{R})(k=1, \ldots, l)$, and $g_{1 k}, g_{2 k}:[a, b] \rightarrow \mathbb{R}$ $(k=1, \ldots, l)$ are continuous functions satisfying the inequalities

$$
\sum_{k=1}^{l} \frac{\left|g_{1 k}(a)\right|}{\nu_{k l}\left(\lambda_{1}\right) \nu_{l n+1}\left(\lambda_{1}\right)}<1, \quad \sum_{k=1}^{l} \frac{\left|g_{2 k}(b)\right|}{\nu_{k l}\left(\lambda_{2}\right) \nu_{l n+1}\left(\lambda_{2}\right)}<1 .
$$

Then for problem (1.4), (1.2 1$),\left(1.2_{2}\right)$ to be uniquely solvable for each $q \in$ $L_{n-1-\lambda_{1}, n-1-\lambda_{2}}(] a, b[; \mathbb{R})$ it is necessary and sufficient that the corresponding homogeneous problem $\left(1.4_{0}\right),\left(1.2_{1}\right),\left(1.2_{2}\right)$ have the trivial solution only.

In the rest of this paragraph it is assumed that $\left.p_{k}:\right] a, b[\rightarrow \mathbb{R}(k=$ $1, \ldots, l)$ are the fixed functions satisfying conditions (2.1), (2.2) and problem $\left(1.4_{0}\right),\left(1.2_{1}\right),\left(1.2_{2}\right)$ has the trivial solution only. Then by Proposition 2.2 problem $(1.4),\left(1.2_{1}\right),\left(1.2_{2}\right)$ has the unique solution for each $q \in$ $L_{n-1-\lambda_{1}, n-1-\lambda_{2}}(] a, b[; \mathbb{R})$. The operator $\mathcal{G}: \mathrm{L}_{n-1-\lambda_{1}, n-1-\lambda_{2}}(] a, b[; \mathbb{R}) \rightarrow$ $\widetilde{\mathbb{C}}_{l o c}^{n-1}(] a, b[; \mathbb{R})$ that puts the solution $u(t)=\mathcal{G}(q)(t)$ of problem $(1.4),\left(1.2_{1}\right)$, $\left(1.2_{2}\right)$ into correspondence with each $q \in L_{n-1-\lambda_{1}, n-1-\lambda_{2}}(] a, b[; \mathbb{R})$ will be called the Green operator of problem $\left(1.4_{0}\right),\left(1.2_{1}\right),\left(1.2_{2}\right)$. 
Proposition 2.4. There exists a positive number $\varrho_{0}$ such that for any $q \in L_{n-1-\lambda_{1}, n-1-\lambda_{2}}(] a, b[; \mathbb{R})$ we have the inequalities

$$
\begin{aligned}
&\left|\frac{d^{k-1} \mathcal{G}(q)(t)}{d t^{k-1}}\right| \leq \varrho_{0} \sigma_{k, \lambda_{1}, \lambda_{2}}(t)|q(\cdot)|_{n-1-\lambda_{1}, n-1-\lambda_{2}} \\
& \text { for } \quad a<t<b \quad(k=1, \ldots, l)
\end{aligned}
$$

and

$$
\begin{gathered}
\left|\frac{d^{n-1} \mathcal{G}(q)(t)}{d t^{n-1}}-\frac{d^{n-1} \mathcal{G}(q)(s)}{d s^{n-1}}\right| \leq \int_{s}^{t} p^{*}(\tau) d \tau+\left|\int_{s}^{t} q(\tau) d \tau\right| \\
\text { for } a<s \leq t<b,
\end{gathered}
$$

where

$$
p^{*}(t)=\varrho_{0}|q(\cdot)|_{n-1-\lambda_{1}, n-1-\lambda_{2}} \sum_{k=1}^{l}\left|p_{k}(t)\right| \sigma_{k, \lambda_{1}, \lambda_{2}}(t) .
$$

Proposition 2.5. Let

$$
\begin{gathered}
q, q_{j} \in L_{n-1-\lambda_{1}, n-1-\lambda_{2}}(] a, b[; \mathbb{R}) \quad(j=1,2, \ldots), \\
\left.\lim _{j \rightarrow+\infty} \int_{\frac{a+b}{2}}^{t} q_{j}(\tau) d \tau=\int_{\frac{a+b}{2}}^{t} q(\tau) d \tau \quad \text { uniformly inside }\right] a, b[
\end{gathered}
$$

and

$$
\limsup _{j \rightarrow+\infty}\left|q_{j}(\cdot)-q(\cdot)\right|_{n-1-\lambda_{1}, n-1-\lambda_{2}}<+\infty .
$$

Then

$$
\left.\lim _{j \rightarrow+\infty} \frac{d^{k-1} \mathcal{G}\left(q_{j}\right)(t)}{d t^{k-1}}=\frac{d^{k-1} \mathcal{G}(q)(t)}{d t^{k-1}} \quad \text { uniformly inside }\right] a, b[.
$$

The proofs of Propositions 2.1-2.5 are given in [14] ${ }^{5}$ (see Lemma 1, Theorems 1 and 5, Corollaries 1 and 4).

To conclude this paragraph let us consider a quasilinear differential equation

$$
u^{(n)}=\sum_{k=1}^{l} p_{k}(t) u^{(k-1)}+q\left(t, u, \ldots, u^{(n-1)}\right),
$$

where $p_{k}: I_{m} \rightarrow \mathbb{R}(k=1, \ldots, l)$ are measurable functions satisfying (2.1), (2.2),

$$
q \in K_{l o c}(] a, b\left[\times \mathbb{R}^{n} ; \mathbb{R}\right) .
$$

\footnotetext{
${ }^{5}$ See also [12] and [13].
} 
Proposition 2.6. Let problem $\left(1.4_{0}\right),\left(1.2_{1}\right),\left(1.2_{2}\right)$ have the trivial solution only. Let, moreover, there exist functions $q_{0} \in L_{n-1-\lambda_{1}, n-1-\lambda_{2}}(] a, b[; \mathbb{R})$ and $q^{*} \in L_{n-1-\lambda_{1}, n-1-\lambda_{2}}(] a, b\left[; \mathbb{R}_{+}\right)$such that the inequality

$$
\left|q\left(t, x_{1}, \ldots, x_{n}\right)-q_{0}(t)\right| \leq q^{*}(t)
$$

is fulfilled on $] a, b\left[\times \mathbb{R}^{n}\right.$. Then problem $(2.3),\left(1.2_{1}\right),\left(1.2_{2}\right)$ is solvable.

Proof. Let $\mathcal{G}$ be the Green operator of problem $\left(1.4_{0}\right),\left(1.2_{1}\right),\left(1.2_{2}\right)$. By Proposition 2.4 there exists $\varrho_{0}>0$ such that the inequalities

$$
\begin{gathered}
\left|\frac{d^{k-1} \mathcal{G}(\widetilde{q})(t)}{d t^{k-1}}\right| \leq \varrho_{0} \sigma_{k, \lambda_{1}, \lambda_{2}}(t)|\widetilde{q}(\cdot)|_{n-1-\lambda_{1}, n-1-\lambda_{2}} \\
\text { for } a<t<b(k=1, \ldots, l)
\end{gathered}
$$

and

$$
\begin{gathered}
\left|\frac{d^{n-1} \mathcal{G}(\widetilde{q})(t)}{d t^{n-1}}-\frac{d^{n-1} \mathcal{G}(\widetilde{q})(s)}{d s^{n-1}}\right| \leq \\
\leq \varrho_{0}|\widetilde{q}(\cdot)|_{n-1-\lambda_{1}, n-1-\lambda_{2}} \sum_{k=1}^{l} \int_{s}^{t}\left|p_{k}(\tau)\right| \sigma_{k, \lambda_{1}, \lambda_{2}}(\tau) d \tau+\left|\int_{s}^{t} \widetilde{q}(\tau) d \tau\right| \\
\text { for } a<s \leq t<b
\end{gathered}
$$

are fulfilled for any $\widetilde{q} \in L_{n-1-\lambda_{1}, n-1-\lambda_{2}}(] a, b[; \mathbb{R})$.

Let $\mathbb{C}^{n-1}(] a, b[; \mathbb{R})$ be a topologic space of $n-1$ times continuously differentiable functions $u:] a, b[\rightarrow \mathbb{R}$, where by the convergence of the sequence $\left(u_{i}\right)_{i=1}^{+\infty}$ we mean the uniform convergence of the sequences $\left(u_{i}^{(k)}\right)_{i=1}^{+\infty}$ $(k=0, \ldots, n-1)$ inside $] a, b[$.

It is assumed that

$$
\begin{gathered}
\varrho=\varrho_{0}\left[\left|q_{0}(\cdot)\right|_{n-1-\lambda_{1}, n-1-\lambda_{2}}+\left|q^{*}(\cdot)\right|_{n-1-\lambda_{1}, n-1-\lambda_{2}}\right], \\
p^{*}(t)=\varrho \sum_{k=1}^{l}\left|p_{k}(t)\right| \sigma_{k, \lambda_{1}, \lambda_{2}}(t)+\left|q_{0}(t)\right|+q^{*}(t),
\end{gathered}
$$

and $A$ is the set of all elements $u$ of the space $\mathbb{C}^{n-1}(] a, b[; \mathbb{R})$ satisfying the inequalities

$$
\begin{aligned}
& \left|u^{(k-1)}(t)\right| \leq \varrho \sigma_{k, \lambda_{1}, \lambda_{2}}(t) \quad \text { for } a<t<b \quad(k=1, \ldots, l), \\
& \left|u^{(n-1)}(t)-u^{(n-1)}(s)\right| \leq \int_{s}^{t} p^{*}(\tau) d \tau \text { for } a<s \leq t<b .
\end{aligned}
$$

It is obvious that $A$ is a convex set. On the other hand, by the Arcela-Askoli lemma it immediately follows that $A$ is a compactum. 
The operator $\widetilde{\mathcal{G}}$ is given on $A$ as follows:

$$
\widetilde{\mathcal{G}}(u)(t)=\mathcal{G}\left(q\left(\cdot, u(\cdot), \ldots, u^{(n-1)}(\cdot)\right)\right)(t) \text { for } u \in A .
$$

According to $(2.5)-(2.7)$, for any $u \in A$ the function $\widetilde{u}(\cdot)=\widetilde{\mathcal{G}}(u)(\cdot)$ satisfies the inequalities

$$
\begin{gathered}
\left|\widetilde{u}^{(k-1)}(t)\right| \leq \varrho_{0}\left|q\left(\cdot, u(\cdot), \ldots, u^{(n-1)}(\cdot)\right)\right|_{n-1-\lambda_{1}, n-1-\lambda_{2}} \sigma_{k, \lambda_{1}, \lambda_{2}}(t) \leq \\
\leq \varrho \sigma_{k, \lambda_{1}, \lambda_{2}}(t) a<t<b \text { for } a<t<b \quad(k=1, \ldots, l), \\
\left|\widetilde{u}^{(n-1)}(t)-\widetilde{u}^{(n-1)}(s)\right| \leq \varrho \sum_{k=1}^{l} \int_{s}^{t}\left|p_{k}(\tau)\right| \sigma_{k, \lambda_{1}, \lambda_{2}}(\tau) d \tau+ \\
+\left|\int_{s}^{t} q\left(\tau, u(\tau), \ldots, u^{(n-1)}(\tau)\right) d \tau\right| \leq \int_{s}^{t} p^{*}(\tau) d \tau \text { for } a<s \leq t<b .
\end{gathered}
$$

Therefore the operator $\widetilde{\mathcal{G}}$ maps $A$ into itself. On the other hand, by Proposition 2.5 condition (2.4) guarantees the continuity of the operator $\widetilde{\mathcal{G}}$. According to the Chauder-Tikhonov theorem [4], $\widetilde{\mathcal{G}}$ has at least one fixed point. Therefore there exists a function $u$ such that $u(t)=\mathcal{G}(q(\cdot, u(\cdot), \ldots$, $\left.\left.u^{(n-1)}(\cdot)\right)\right)(t)$ for $a<t<b$. Hence it is clear that $u$ is the solution of problem (2.3), (1.2 1$),\left(1.2_{2}\right)$.

\section{$\S$ 3. Proof of the Main Results}

Proof of Theorem 1.1. Let $\rho_{0}$ be a positive number for which Proposition 2.1 is valid. According to (1.9) there exists $\rho^{*}>0$ such that

$$
l \rho_{0}\left[|q(\cdot, \rho)|_{n-1-\lambda_{1}, n-1-\lambda_{2}}+|q(\cdot)|_{n-1-\lambda_{1}, n-1-\lambda_{2}}\right]<\rho \text { for } \rho \geq \rho^{*} .
$$

Let

$$
\chi(s)= \begin{cases}1 & \text { for } 0 \leq s \leq \rho^{*}, \\ 2-\frac{s}{\rho^{*}} & \text { for } \rho^{*} \leq s \leq 2 \rho^{*}, \\ 0 & \text { for } s \geq 2 \rho^{*}\end{cases}
$$

and $\varepsilon_{0}=\frac{a+b}{4}$. Assume that for each $j$

$$
\begin{gathered}
\varepsilon_{j}(t)= \begin{cases}0 & \text { for } t \in] a, a+\frac{\varepsilon_{0}}{j}[\cup] b-\frac{\varepsilon_{0}}{j}, b[, \\
1 & \text { for } t \in\left[a+\frac{\varepsilon_{0}}{j}, b-\frac{\varepsilon_{0}}{j}\right],\end{cases} \\
q_{j}\left(t, x_{1}, \ldots, x_{n}\right)=\varepsilon_{j}(t) \chi\left(\sum_{i=1}^{l} \frac{\left|x_{i}\right|}{\sigma_{i, \lambda_{1}, \lambda_{2}}(t)}\right)\left[f\left(t, x_{1}, \ldots, x_{n}\right)-\right.
\end{gathered}
$$




$$
\left.-\sum_{k=1}^{l} p_{1 k}(t) x_{k}-q_{0}(t)\right]+q_{0}(t)
$$

and consider the differential equation

$$
u^{(n)}=\sum_{k=1}^{l} p_{1 k}(t) u^{(k-1)}+q_{j}\left(t, u, \ldots, u^{(n-1)}\right)
$$

for an arbitrary natural number $j$.

Taking into account inequalities (1.6), (1.7), from (3.2)-(3.4) we obtain on $] a, b\left[\times \mathbb{R}^{n}\left|q_{j}\left(t, x_{1}, \ldots, x_{n}\right)-q_{0}(t)\right| \leq q_{j}^{*}(t)\right.$, where

$$
q_{j}^{*}(t)=\varepsilon_{j}(t) q\left(t, 2 \rho^{*}\right)+2 \rho^{*} \varepsilon_{j}(t) \sum_{k=1}^{l}\left|p_{2 k}(t)-p_{1 k}(t)\right| \sigma_{k, \lambda_{1}, \lambda_{2}}(t) .
$$

By (1.8) and (3.3) it is easy to ascertain that $q_{j}^{*} \in L\left([a, b] ; \mathbb{R}_{+}\right)$. Therefore, according to Proposition 2.6, Problem (3.5), $\left(1.2_{1}\right),\left(1.2_{2}\right)$ has a solution $u_{j}$.

By (3.4) and (3.5) it is obvious that

$$
u_{j}^{(n)}(t)=\sum_{k=1}^{l} \widetilde{p}_{k j}(t) u_{j}^{(k-1)}(t)+\widetilde{q}_{j}(t),
$$

where

$$
\begin{aligned}
\widetilde{p}_{k j}(t) & =p_{1 k}(t)+\varepsilon_{j}(t) \chi\left(\sum_{i=1}^{l} \frac{\left|u_{j}^{(i-1)}(t)\right|}{\sigma_{i, \lambda_{1}, \lambda_{2}}(t)}\right) \times \\
& \times\left[p_{k}\left(t, u_{j}(t), \ldots, u_{j}^{(n-1)}(t)\right)-p_{1 k}(t)\right], \\
\widetilde{q}_{k j}(t) & =\varepsilon_{j}(t) \chi\left(\sum_{i=1}^{l} \frac{\left|u_{j}^{(i-1)}(t)\right|}{\sigma_{i, \lambda_{1}, \lambda_{2}}(t)}\right)\left[f\left(t, u_{j}(t), \ldots, u_{j}^{(n-1)}(t)\right)-\right. \\
& \left.-\sum_{k=1}^{l} p_{k}\left(t, u_{j}(t), \ldots, u_{j}^{(n-1)}(t)\right) u_{j}^{(k-1)}(t)-q_{0}(t)\right]+q_{0}(t) .
\end{aligned}
$$

On the other hand, (1.6) and (1.7) imply

$$
p_{1 k}(t) \leq \widetilde{p}_{k j}(t) \leq p_{2 k}(t) \text { for } a<t<b \quad(k=1, \ldots, l)
$$

and

$$
\left|\widetilde{q}_{j}(t)-q_{0}(t)\right| \leq q\left(t, \rho_{j}\right) \text { for } a<t<b,
$$

where

$$
\rho_{j}=\operatorname{vraimax}\left\{\sum_{i=1}^{l} \frac{\left|u_{j}^{(i-1)}(t)\right|}{\sigma_{i, \lambda_{1}, \lambda_{2}}(t)}: a<t<b\right\}
$$


In view of conditions (1.8), (3.7) and the choice of the number $\rho_{0}$ we have $\left|u_{j}^{(i-1)}(t)\right| \leq \rho_{0}\left|\widetilde{q}_{j}(\cdot)\right|_{n-1-\lambda_{1}, n-1-\lambda_{2}} \sigma_{i, \lambda_{1}, \lambda_{2}}(t)$ for $a<t<b \quad(i=1, \ldots, l)$.

Hence, taking into account (3.8) and (3.9), we find

$$
\rho_{j} \leq l \rho_{0}\left[\left|q_{0}(\cdot)\right|_{n-1-\lambda_{1}, n-1-\lambda_{2}}+\left|q\left(\cdot, \rho_{j}\right)\right|_{n-1-\lambda_{1}, n-1-\lambda_{2}}\right] .
$$

Consequently, by (3.1) $\rho_{j} \leq \rho^{*}$. According to this inequality, for each $j$ (3.2), (3.6)-(3.9) imply

$$
\begin{gathered}
\chi\left(\sum_{i=1}^{l} \frac{\left|u_{j}^{(i-1)}(t)\right|}{\sigma_{i, \lambda_{1}, \lambda_{2}}(t)}\right)=1 \text { for } a<t<b, \\
\left|\widetilde{q}_{j}(t)-q_{0}(t)\right| \leq q\left(t, \rho^{*}\right) \text { for } a<t<b, \\
\left|u_{j}^{(k-1)}(t)\right| \leq \rho^{*} \sigma_{k, \lambda_{1}, \lambda_{2}}(t) \text { for } a<t<b(k=1, \ldots, l)
\end{gathered}
$$

and

$$
\left|u_{j}^{(n)}(t)-q_{0}(t)\right| \leq p^{*}(t) \text { for } a<t<b,
$$

where

$$
p^{*}(t)=\rho^{*} \sum_{k=1}^{l}\left(\left|p_{1 k}(t)\right|+\left|p_{2 k}(t)\right|\right) \sigma_{k, \lambda_{1}, \lambda_{2}}(t)+q\left(t, \rho^{*}\right)
$$

belongs to the class $L_{l o c}(] a, b\left[; \mathbb{R}_{+}\right)$.

By virtue of $(3.12)$ and $(3.13)$ the sequences $\left(u_{j}^{(k-1)}\right)_{j=1}^{+\infty}(k=1, \ldots, n)$ are uniformly bounded and equicontinuous inside $] a, b[$. Therefore by the Arcela-Askoli lemma it can be assumed without loss of generality that they converge uniformly inside $] a, b[$.

Let $u(t)=\lim _{j \rightarrow+\infty} u_{j}(t)$ for $a<t<b$. Then

$$
u^{(k-1)}(t)=\lim _{j \rightarrow+\infty} u_{j}^{(k-1)}(t) \text { for } a<t<b \quad(k=1, \ldots, n) .
$$

Taking into account (3.3), (3.6), (3.10), and (3.13), it readily follows from (3.14) that $u$ is a solution of equation (1.1).

On the other hand, since the sequences $\left(u_{j}^{(k-1)}\right)_{j=1}^{+\infty}(k=1, \ldots, n)$ are uniformly bounded inside $] a, b[$ and on account of (1.8), (3.11), (3.12) and the equalities $u_{j}^{(k-1)}(a)=0\left(k=1, \ldots, n_{1}\right), u_{j}^{(k-1)}(b)=0\left(k=1, \ldots, n_{m}\right)$, from (3.6) we find

$$
\begin{gathered}
\left|u_{j}^{(k-1)}(t)\right| \leq r_{1}(t-a)^{\lambda_{1}-k+1}+\int_{a}^{t}(t-s)^{n_{1}-k} \int_{s}^{\frac{a+b}{2}}(\tau-s)^{n-n_{1}-1} \widetilde{p}(\tau) d \tau d s \\
\quad \text { for } a<t \leq \frac{a+b}{2}, \quad l \in\left\{1, \ldots, n_{1}\right\} \quad\left(k=1, \ldots, n_{1}\right)
\end{gathered}
$$


and

$$
\begin{gathered}
\left|u_{j}^{(k-1)}(t)\right| \leq r_{2}(b-t)^{\lambda_{2}-k+1}+\int_{t}^{b}(s-t)^{n_{m}-k} \int_{\frac{a+b}{2}}^{s}(s-\tau)^{n-n_{m}-1} \widetilde{p}(\tau) d \tau d s \\
\text { for } \frac{a+b}{2} \leq t<b, \quad l \in\left\{1, \ldots, n_{m}\right\} \quad\left(k=1, \ldots, n_{m}\right),
\end{gathered}
$$

where $\widetilde{p}(t)=p^{*}(t)-q\left(t, \rho^{*}\right)$, and $r_{1}$ and $r_{2}$ are positive numbers not depending on $j$. Taking into account these estimates together with (3.12) and (3.14), we ascertain that $u$ satisfies the boundary conditions $\left(1.2_{1}\right),\left(1.2_{2}\right)$.

Thus $u$ is the solution of problem $(1.1),\left(1.2_{1}\right),\left(1.2_{2}\right)$.

Proof of Corollary 1.1. We set

$$
\begin{aligned}
& \gamma\left(t, x_{1}, \ldots, x_{n}\right)=\left[f\left(t, x_{1}, \ldots, x_{n}\right)-q_{0}(t)\right] \times \\
& \times\left[1+\sum_{k=1}^{l} p_{k}(t)\left|x_{k}\right|+q\left(t, \sum_{i=1}^{l} \frac{\left|x_{i}\right|}{\sigma_{i, \lambda_{1}, \lambda_{2}}(t)}\right)\right]^{-1}
\end{aligned}
$$

and $\widetilde{p}_{k}\left(t, x_{1}, \ldots, x_{n}\right)=\gamma\left(t, x_{1}, \ldots, x_{n}\right) p_{k}(t) \operatorname{sign} x_{k}(k=1, \ldots, l)$. Then

$$
\begin{gathered}
f\left(t, x_{1}, \ldots, x_{n}\right)=\sum_{k=1}^{l} \widetilde{p}_{k}\left(t, x_{1}, \ldots, x_{n}\right) x_{k}+ \\
+\left[1+q\left(t, \sum_{i=1}^{l} \frac{\left|x_{i}\right|}{\sigma_{i, \lambda_{1}, \lambda_{2}}(t)}\right) \gamma\left(t, x_{1}, \ldots, x_{n}\right)\right]+q_{0}(t)
\end{gathered}
$$

and

$$
\widetilde{p}_{k} \in K_{l o c}^{0}\left(I_{m} \times \mathbb{R}^{n} ; \mathbb{R}\right) .
$$

On the other hand, according to (1.9) and (1.10), on $] a, b\left[\times \mathbb{R}^{n}\right.$ we have

$$
\begin{gathered}
\left|\gamma\left(t, x_{1}, \ldots, x_{n}\right)\right|<1 \\
-p_{k}(t) \leq \widetilde{p}_{k}\left(t, x_{1}, \ldots, x_{n}\right) \leq p_{k}(t) \quad(k=1, \ldots, l)
\end{gathered}
$$

and

$$
\begin{aligned}
& \mid f\left(t, x_{1}, \ldots, x_{n}\right)-\sum_{k=1}^{l} \widetilde{p}_{k}\left(t, x_{1}, \ldots, x_{n}\right) x_{k}-q_{0}(t) \mid \leq \\
& \leq \widetilde{q}\left(t, \sum_{i=1}^{l} \frac{\left|x_{i}\right|}{\sigma_{i, \lambda_{1}, \lambda_{2}}(t)}\right),
\end{aligned}
$$


where the function $\widetilde{q}(t, \rho)=1+q(t, \rho)$ satisfies the condition

$$
\lim _{\rho \rightarrow+\infty} \frac{|\widetilde{q}(\cdot, \rho)|_{n-1-\lambda_{1}, n-1-\lambda_{2}}}{\rho}=0 .
$$

By Theorem 1.1 from conditions (1.11), (3.15)-(3.18) we conclude that problem $(1.1),\left(1.2_{1}\right),\left(1.2_{2}\right)$ is solvable.

Proof of Theorem 1.2. By the inequalities (1.12), the equality

$$
f\left(t, x_{1}, \ldots, x_{l}\right)=\sum_{k=1}^{l} p_{k}\left(t, x_{1}, \ldots, x_{l}\right) x_{k}+q_{0}(t),
$$

where

$$
\begin{aligned}
p_{k}\left(t, x_{1}, \ldots, x_{l}\right)= & \int_{0}^{1} \frac{\partial f\left(t, \xi x_{1}, \ldots, \xi x_{l}\right)}{\partial x_{k}} d \xi \quad(k=1, \ldots, l), \\
& q_{0}(t)=f(t, 0, \ldots, 0)
\end{aligned}
$$

yields

$$
\left|f\left(t, x_{1}, \ldots, x_{l}\right)-\sum_{k=1}^{l} p_{k}\left(t, x_{1}, \ldots, x_{l}\right) x_{k}-q_{0}(t)\right|=0
$$

and $p_{1 k}(t) \leq p_{k}\left(t, x_{1}, \ldots, x_{l}\right) \leq p_{2 k}(t)(k=1, \ldots, l)$. Therefore by Theorem 1.1 problem $\left(1.1^{\prime}\right),\left(1.2_{1}\right),\left(1.2_{2}\right)$ has a solution $u_{0}$.

Let us show that $u_{0}$ is the unique solution of this problem. Assume that $u_{1}$ is an arbitrary solution of problem $\left(1.1^{\prime}\right),\left(1.2_{1}\right),\left(1.2_{2}\right)$. Set

$$
\begin{gathered}
u(t)=u_{0}(t)-u_{1}(t), \quad \widetilde{p}_{k}\left(t ; x_{1}, \ldots, x_{l} ; y_{1}, \ldots, y_{l}\right)= \\
=\int_{0}^{1} \frac{\partial f\left(t, \xi x_{1}+(1-\xi) y_{1}, \ldots, \xi x_{l}+(1-\xi) y_{l}\right)}{\partial x_{k}} d \xi
\end{gathered}
$$

and $p_{k}(t)=\widetilde{p}_{k}\left(t ; u_{0}(t), \ldots, u_{0}^{(l-1)}(t) ; u_{1}(t), \ldots, u_{1}^{(l-1)}(t)\right)$.

By virtue of the equality

$$
f\left(t, x_{1}, \ldots, x_{l}\right)-f\left(t, y_{1}, \ldots, y_{l}\right)=\sum_{k=1}^{l} \widetilde{p}_{k}\left(t ; x_{1}, \ldots, x_{l} ; y_{1}, \ldots, y_{l}\right)\left(x_{k}-y_{k}\right)
$$

and the inequalities (1.12), $u$ is a solution of the equation

$$
u^{(n)}=\sum_{k=1}^{l} p_{k}(t) u^{(k-1)}
$$


satisfying the boundary conditions $\left(1.2_{1}\right),\left(1.2_{2}\right)$ and

$$
p_{1 k}(t) \leq p_{k}(t) \leq p_{2 k}(t) \text { for } a<t<b \quad(k=1, \ldots, l) .
$$

But by (1.8) and (3.20) problem (3.19), $\left(1.2_{1}\right),\left(1.2_{2}\right)$ has the trivial solution only, i.e., $u_{1}(t) \equiv u_{0}(t)$.

Proof of Theorem 1.3. Set

$$
p(t)=\frac{r(t)}{\sigma_{1, \lambda_{1}, \lambda_{2}}(t)} \quad \text { for } a<t<b .
$$

Let us show that

$$
(p) \in S^{+}\left(a, b ; n, n_{1} ; \lambda_{1}\right) \cap S^{-}\left(a, b ; n, n_{m} ; \lambda_{2}\right) .
$$

By virtue of $\left(1.14_{1}\right)$ there exist points $t_{0 n_{1}}, t_{0 n_{1}+1}, \ldots, t_{0 n-1}$ such that

$$
a<t_{0 n_{1}} \leq \cdots \leq t_{0 n-1}<b
$$

and

$$
u_{0}^{(k)}(r)\left(t_{0 k}\right)=0 \quad\left(k=n_{1}, \ldots, n-1\right)
$$

At the same time, $u_{0}^{(n)}(r)(t)=r(t) \geq 0$ for $a<t<b$. Therefore by (3.22) it is obvious that for each $k \in\left\{n_{1}, \ldots, n-1\right\}$ the function $u_{0}^{(k)}(r)(\cdot)$ does not change its sign on $] a, t_{0 k}[$ and, taking into account (3.23), we readily obtain

$$
\begin{gathered}
\left|u_{0}^{\left(n_{1}\right)}(r)(t)\right| \geq \frac{1}{\left(n-n_{1}-1\right) !} \int_{t}^{t_{0 n_{1}}}(\tau-t)^{n-n_{1}-1} r(\tau) d \tau \\
\text { for } a<t \leq t_{0 n_{1}} .
\end{gathered}
$$

At the same time, by $\left(1.14_{1}\right)$ we have

$$
\begin{aligned}
\left|u_{0}(r)(t)\right|= & \frac{1}{\left(n_{1}-1\right) !} \int_{a}^{t}(\tau-t)^{n_{1}-1}\left|u^{\left(n_{1}\right)}(r)(\tau)\right| d \tau \\
& \text { for } a<t \leq \min \left\{t_{2}, t_{0 n_{1}}\right\} .
\end{aligned}
$$

Using (1.16), choose $\alpha \in] a, t_{2}\left[\right.$ such that $\alpha \leq t_{0 n_{1}}$ and

$$
\rho_{\lambda_{1}, \lambda_{2}}(r)<\frac{(b-\alpha)^{\lambda_{2}} \prod_{i=2}^{m-1}\left|\alpha-t_{i}\right|^{n_{i}}}{(b-a)^{\lambda_{2}} \prod_{i=2}^{m-1}\left|a-t_{i}\right|^{n_{i}}} .
$$


By (3.24) the latter equality yields

$$
\begin{gathered}
\left|u_{0}(r)(t)\right| \geq \frac{(b-\alpha)^{\lambda_{2}} \prod_{i=2}^{m-1}\left|\alpha-t_{i}\right|^{n_{i}}}{\left(n_{1}-1\right) !\left(n-n_{1}-1\right) !} \times \\
\times \int_{a}^{t}(t-s)^{n_{1}-1} \int_{s}^{\alpha}(\tau-t)^{n-n_{1}-1}(\tau-a)^{\lambda_{1}}|p(\tau)| d \tau d s \\
\text { for } a<t \leq \alpha,
\end{gathered}
$$

whence

$$
\begin{gathered}
\rho_{\lambda_{1}, \lambda_{2}}(r) \geq \limsup _{t \rightarrow a} \frac{\left|u_{0}(r)(t)\right|}{\sigma_{1, \lambda_{1}, \lambda_{2}}(t)} \geq \\
\geq \frac{(b-\alpha)^{\lambda_{2}} \prod_{i=2}^{m-1}\left|\alpha-t_{i}\right|^{n_{i}}}{(b-a)^{\lambda_{2}} \prod_{i=2}^{m-1}\left|a-t_{i}\right|^{n_{i}}} \limsup _{t \rightarrow a} \frac{(t-a)^{-\lambda_{1}}}{\left(n_{1}-1\right) !\left(n-n_{1}-1\right) !} \times \\
\times \int_{a}^{t}(t-s)^{n_{1}-1} \int_{s}^{\alpha}(\tau-t)^{n-n_{1}-1}(\tau-a)^{\lambda_{1}}|p(\tau)| d \tau d s .
\end{gathered}
$$

Therefore by virtue of (3.25) we obtain

$$
\begin{gathered}
\limsup _{t \rightarrow a} \frac{(t-a)^{-\lambda_{1}}}{\left(n_{1}-1\right) !\left(n-n_{1}-1\right) !} \times \\
\times \int_{a}^{t}(t-s)^{n_{1}-1} \int_{s}^{\alpha}(\tau-t)^{n-n_{1}-1}(\tau-a)^{\lambda_{1}}|p(\tau)| d \tau d s<1 .
\end{gathered}
$$

The inequality

$$
\begin{gathered}
\limsup _{t \rightarrow b} \frac{(b-t)^{-\lambda_{2}}}{\left(n_{m}-1\right) !\left(n-n_{m}-1\right) !} \times \\
\times \int_{t}^{b}(s-t)^{n_{m}-1} \int_{\beta}^{s}(s-\tau)^{n-n_{m}-1}(b-\tau)^{\lambda_{2}}|p(\tau)| d \tau d s<1,
\end{gathered}
$$

where $\beta \in] t_{m-1}, b[$, is proved in a similar manner. Thus (3.21) is valid.

Now let us show that

$$
(-p, p) \in V\left(t_{1}, \ldots, t_{m} ; n_{1}, \ldots, n_{m} ; \lambda_{1}, \lambda_{2}\right) .
$$

Due to (3.21) it it is sufficient to verify that under the boundary conditions $\left(1.14_{1}\right),\left(1.14_{2}\right)$ the equation

$$
u^{(n)}=\widetilde{p}(t) u
$$


has the trivial solution only for any function $\widetilde{p}:] a, b[\rightarrow \mathbb{R}$ satisfying the conditions $\sigma_{1, \lambda_{1}, \lambda_{2}}(\cdot) \widetilde{p}(\cdot) \in L_{l o c}(] a, b[; \mathbb{R})$ and

$$
-p(t) \leq \widetilde{p}(t) \leq p(t) \text { for } a<t<b .
$$

Let $u$ be an arbitrary solution of problem $(3.27),\left(1.14_{1}\right),\left(1.14_{2}\right)$ and $g(\cdot, \cdot)$ be the Green function of the equation

$$
u^{(n)}=0
$$

by the boundary conditions of de la Vallée-Poussin (1.14 $)$. According to the Chichkin theorem $[3]^{6} g(t, \tau)\left(t-t_{1}\right)^{n_{1}} \cdots\left(t-t_{m}\right)^{n_{m}} \geq 0$ for $a \leq t, \tau \leq b$. Therefore

$$
\left|u_{0}(r)(t)\right|=\int_{a}^{b}|g(t, \tau)| r(\tau) d \tau .
$$

On account of (3.28), (3.30) the equality

$$
u(t)=\int_{a}^{b} g(t, \tau) \widetilde{p}(\tau) u(\tau) d \tau
$$

yields $\gamma \leq \gamma \rho_{\lambda_{1}, \lambda_{2}}(r)$, where

$$
\gamma=\text { vrai } \max \left\{\frac{|u(t)|}{\sigma_{1, \lambda_{1}, \lambda_{2}}(t)}: a<t<b\right\} .
$$

Hence, taking into account (1.16), we obtain $\gamma=0$, i.e., $u(t) \equiv 0$. Therefore (3.26) holds. Now by virtue of (1.15) we conclude that all the conditions of Corollary 1.1 are fulfilled. Therefore problem $(1.13),\left(1.14_{1}\right),\left(1.14_{2}\right)$ is solvable.

Proof of Corollary 1.2. We introduce the notation

$$
\begin{gathered}
\eta\left(a, b ; n_{1}, n_{2} ; \lambda_{1}, \lambda_{2}\right)= \\
=\left[n_{1} ! n_{2} !\left(\lambda_{1}-n_{1}+1\right)\left(\lambda_{2}-n_{2}+1\right)\left(n_{1}-\lambda_{1}\right)\left(n_{2}-\lambda_{2}\right)(b-a)^{n}\right] \times \\
\times\left[2 n_{1}\left(\lambda_{2}-n_{2}+1\right)\left(n_{1}-\lambda_{1}\right)\left(\lambda_{1}-n_{1}+1+n_{2}-\lambda_{2}\right)+\right. \\
\left.+2 n_{2}\left(\lambda_{1}-n_{1}+1\right)\left(n_{2}-\lambda_{2}\right)\left(\lambda_{2}-n_{2}+1+n_{1}-\lambda_{1}\right)\right]^{-1}, \\
r(t)=\frac{r_{0}}{(t-a)^{n-\lambda_{1}}(b-t)^{n-\lambda_{2}}} .
\end{gathered}
$$

Now condition (1.17) can be written as

$$
r_{0}<\eta\left(a, b ; n_{1}, n_{2} ; \lambda_{1}, \lambda_{2}\right) .
$$

${ }^{6}$ See also [9], Lemma 4.2 . 
By Theorem 1.3, to prove the corollary it is sufficient to show that

$$
\rho_{\lambda_{1}, \lambda_{2}}(r)=\sup \left\{\frac{\left|u_{0}(r)(t)\right|}{(t-a)^{\lambda_{1}}(b-t)^{\lambda_{2}}}: a<t<b\right\}<1,
$$

where $u_{0}(r)(\cdot)$ is the solution of the equation $u^{(n)}=r(t)$, satisfying the boundary conditions $\left(1.14_{1}\right)$ for $m=2$.

As shown in [1], for $m=2$ the Green function $g(\cdot, \cdot)$ of problem (3.29), $\left(1.14_{1}\right)$ admits the estimate

$$
\begin{aligned}
|g(t, \tau)| \leq \frac{(t-a)^{n_{1}-1}(b-t)^{n_{2}-1}(\tau-a)^{n_{2}-1}(b-\tau)^{n_{1}-1}}{\left(n_{1}-1\right) !\left(n_{2}-1\right) !(b-a)^{n-1}} \times \\
\times\left\{\begin{array}{ll}
\frac{(t-a)(b-\tau)}{n} & \text { for } t \leq \tau \\
\frac{(b-t)(\tau-a)}{n_{2}} & \text { for } t<\tau
\end{array} .\right.
\end{aligned}
$$

Therefore from the equality

$$
u_{0}(r)(t)=\int_{a}^{b} g(t, \tau) r(\tau) d \tau
$$

we find

$$
\left|u_{0}(r)(t)\right| \leq \frac{r_{0}}{\eta\left(a, b ; n_{1}, n_{2} ; \lambda_{1}, \lambda_{2}\right)}(t-a)^{\lambda_{1}}(b-t)^{\lambda_{2}},
$$

whence by (3.31) we obtain

$$
\rho_{\lambda_{1}, \lambda_{2}}(r)<1
$$

Proof of Corollary 1.3. Let

$$
\begin{aligned}
r(t) & =r_{0}(t-a)^{\lambda_{1}}(b-t)^{\lambda_{2}}\left[\frac{\lambda_{1}\left(1-\lambda_{1}\right)}{(t-a)^{2}}+\right. \\
& \left.+\frac{2 \lambda_{1} \lambda_{2}}{(t-a)(b-t)}+\frac{\lambda_{2}\left(1-\lambda_{2}\right)}{(b-t)^{2}}\right] .
\end{aligned}
$$

Clearly, $r \in L_{1-\lambda_{1}, 1-\lambda_{2}}(] a, b\left[; \mathbb{R}_{+}\right)$. On the other hand, it is not difficult to verify that the function $u_{0}(r)(t)=-r_{0}(t-a)^{\lambda_{1}}(b-t)^{\lambda_{2}}$ is the solution of the problem

$$
u^{\prime \prime}=r(t), \quad u(a)=u(b)=0 .
$$

By condition (1.25) we obtain $\rho_{\lambda_{1}, \lambda_{2}}(r)=r_{0}<1$. Thus all the conditions of Theorem 1.3 are fulfilled. 
Proof of Corollary 1.4. Let

$$
\begin{aligned}
r(t) & =r_{0}(t-a)^{\lambda_{1}}\left(t_{0}-t\right)(b-t)^{\lambda_{2}}\left[\frac{\lambda_{1}\left(1-\lambda_{1}\right)\left(2-\lambda_{1}\right)}{(t-a)^{3}}-\frac{3 \lambda_{1}\left(1-\lambda_{1}\right)}{(t-a)^{2}\left(t-t_{0}\right)}+\right. \\
& +\frac{3 \lambda_{1} \lambda_{2}\left(1-\lambda_{1}\right)}{(t-a)^{2}(b-t)}-\frac{6 \lambda_{1} \lambda_{2}}{(t-a)\left(t-t_{0}\right)(b-t)}-\frac{3 \lambda_{1} \lambda_{2}\left(1-\lambda_{2}\right)}{(t-a)(b-t)^{2}}- \\
& \left.-\frac{3 \lambda_{2}\left(1-\lambda_{2}\right)}{\left(t-t_{0}\right)(b-t)^{2}}-\frac{\lambda_{2}\left(1-\lambda_{2}\right)\left(2-\lambda_{2}\right)}{(b-t)^{3}}\right] .
\end{aligned}
$$

Clearly, $r \in L_{2-\lambda_{1}, 2-\lambda_{2}}(] a, b[; \mathbb{R})$. On the other hand, it is not difficult to verify that the function $u_{0}(r)(t)=-r_{0}(t-a)^{\lambda_{1}}\left(t-t_{0}\right)(b-t)^{\lambda_{2}}$ is the solution of the problem

$$
u^{\prime \prime \prime}=r(t), \quad u(a)=u\left(t_{0}\right)=u(b)=0 .
$$

By condition (1.25) we obtain $\rho_{\lambda_{1}, \lambda_{2}}(r)=r_{0}<1$. Thus all the conditions of Theorem 1.3 are fulfilled.

Proof of Theorem 1.4. As we have ascertained in proving Theorem 1.3, condition (1.16) guarantees the fulfilment of condition (3.26), where

$$
p(t)=\frac{r(t)}{\sigma_{1, \lambda_{1}, \lambda_{2}}(t)} .
$$

Therefore, according to Theorem 1.2, problem $(1.13),\left(1.14_{1}\right),\left(1.14_{2}\right)$ has the unique solution $u$.

From the conditions of the theorem we have

$$
f(\cdot, \widetilde{u}(\cdot)) \in L_{n-1-\lambda_{1}, n-1-\lambda_{2}}(] a, b[; \mathbb{R})
$$

for any $\widetilde{u} \in \mathbb{C}_{l o c}^{n-1}(] a, b[; \mathbb{R})$ satisfying the boundary conditions $\left(1.14_{1}\right)$, $\left(1.14_{2}\right)$. Therefore, by Proposition 2.3, for each natural number $j$ problem $(1.24),\left(1.14_{1}\right),\left(1.14_{2}\right)$ has the unique solution $u_{j}$.

It is assumed that for each $j$

$$
v_{j}(t)=u_{j}(t)-u(t) .
$$

Clearly, $v_{j}$ satisfies the boundary conditions $\left(1.14_{1}\right),\left(1.14_{2}\right)$,

$$
\gamma_{j}=\operatorname{vraimax}\left\{\frac{\left|v_{j}(t)\right|}{\sigma_{1, \lambda_{1}, \lambda_{2}}(t)}: a<t<b\right\}<+\infty
$$

and

$$
v_{j}^{(n)}=f\left(t, u_{j-1}(t)\right)-f(t, u(t)) .
$$

Repeating the reasoning from the proof of Theorem 1.3 and using the Chichkin theorem, by virtue of $(1.22),(3.32)-(3.34)$ we obtain

$$
\gamma_{j} \leq \gamma_{j-1} \rho_{\lambda_{1}, \lambda_{2}}(r)
$$


Hence $\gamma_{j} \leq \gamma_{1} \rho_{\lambda_{1}, \lambda_{2}}^{j-1}(r)(j=1,2, \ldots)$. Therefore by (1.16), (3.32), (3.33) we obtain (1.23).

Corollaries $1.5-1.7$ are proved similarly to Corollaries $1.2-1.4$, the only difference being that Theorem 1.4 is used instead of Theorem 1.3.

Remark 3.1. In Corollaries 1.3, 1.4, 1.6, and 1.7 condition (1.25) cannot be replaced by equality (1.26), since for $\left.\lambda_{1} \in\right] \frac{1}{2}, 1\left[\right.$ problem (1.18), $\left(1.19_{1}\right)$, $\left(1.19_{2}\right)$, where

$$
f(t, x)=-\frac{\lambda_{1}\left(1-\lambda_{1}\right)}{(t-a)^{2}} x+(t-a)^{\lambda_{1}-2},
$$

and problem $(1.20),\left(1.21_{1}\right),\left(1.21_{2}\right)$, where

$$
f(t, x)=\frac{\lambda_{1}\left(1-\lambda_{1}\right)\left(2-\lambda_{1}\right)}{(t-a)^{3}} x+(t-a)^{\lambda_{1}-3},
$$

have no solutions though all the conditions of these corollaries are fulfilled with the exception of condition (1.25) which is replaced by (1.26).

Proof of Theorem 1.5. Let us assume that the theorem is not true. Then there exist $\varepsilon \in] 0, r[, \alpha \in] a, t_{2}[, \beta \in] t_{m-1}, b\left[,\left(x_{10}, \ldots, x_{l 0}\right) \in \mathcal{D}_{\lambda_{1}, \lambda_{2}}\left(u_{0} ; r\right)\right.$, $\omega \in M\left([\alpha, \beta] \times \mathbb{R}_{+} ; \mathbb{R}_{+}\right)$and a sequence of functions $\eta_{i} \in K_{\text {loc }}\left(I_{m} \times \mathbb{R}^{l} ; \mathbb{R}\right)$ such that

$$
\begin{gathered}
\left|\int_{\alpha}^{t} \eta_{i}\left(\tau, \sigma_{1, \lambda_{1}, \lambda_{2}}(\tau) x_{1}, \ldots, \sigma_{1, \lambda_{1}, \lambda_{2}}(\tau) x_{l}\right) d \tau\right| \leq \frac{1}{i} \\
\text { for } \alpha \leq t \leq \beta, \quad\left(x_{1}, \ldots, x_{l}\right) \in \mathcal{D}_{\lambda_{1}, \lambda_{2}}\left(u_{0} ; r\right), \\
\mid \eta_{i}\left(t, \sigma_{1, \lambda_{1}, \lambda_{2}}(t) x_{1}, \ldots, \sigma_{l, \lambda_{1}, \lambda_{2}}(t) x_{l}\right)- \\
-\eta_{i}\left(t, \sigma_{1, \lambda_{1}, \lambda_{2}}(t) y_{1}, \ldots, \sigma_{l, \lambda_{1}, \lambda_{2}}(t) y_{l} \mid \leq \omega\left(\sum_{k=1}^{l}\left|x_{k}-y_{k}\right|\right)\right. \\
\text { for } \alpha \leq t \leq \beta, \quad\left(x_{1}, \ldots, x_{l}\right) \text { and }\left(y_{1}, \ldots, y_{l}\right) \in \mathcal{D}_{\lambda_{1}, \lambda_{2}}\left(u_{0} ; r\right), \\
(t-a)^{n-1-\lambda_{1}}\left[\int_{t}^{\alpha} \eta_{\lambda_{1}, \lambda_{2}, i}^{*}\left(\tau ; u_{0} ; r\right) d \tau+\left|\int_{t}^{\alpha} \eta_{0 i}(\tau), d \tau\right|\right] \leq \frac{1}{i} \\
(b-t)^{n-1-\lambda_{2}}\left[\int_{\beta}^{t} \eta_{\lambda_{1}, \lambda_{2}, i}^{*}\left(\tau ; u_{0} ; r\right) d \tau+\left|\int_{\beta}^{t} \eta_{0 i}(\tau), d \tau\right|\right] \leq \frac{1}{i} \\
\text { for } \beta \leq t<b,
\end{gathered}
$$


where

$$
\begin{gathered}
\eta_{0 i}(t)=\eta_{i}\left(t, \sigma_{1, \lambda_{1}, \lambda_{2}}(t) x_{10}, \ldots, \sigma_{l, \lambda_{1}, \lambda_{2}}(t) x_{l 0}\right), \\
\eta_{\lambda_{1}, \lambda_{2}, i}^{*}\left(t ; u_{0} ; r\right)= \\
=\sup \left\{\left|\eta_{i}\left(t, \sigma_{1, \lambda_{1}, \lambda_{2}}(t) x_{1}, \ldots, \sigma_{l, \lambda_{1}, \lambda_{2}}(t) x_{l}\right)-\eta_{0 i}\right|:\right. \\
\left.\left(x_{1}, \ldots, x_{l}\right) \in \mathcal{D}_{\lambda_{1}, \lambda_{2}}\left(u_{0} ; r\right)\right\},
\end{gathered}
$$

and for each $i$ the equation

$$
u^{(n)}=f\left(t, u, \ldots, u^{(l-1)}\right)+\eta_{i}\left(t, u, \ldots, u^{(l-1)}\right)
$$

either has no solution contained in $W_{\lambda_{1}, \lambda_{2}}\left(u_{0} ; r\right)$ or has at least one solution contained in $W_{\lambda_{1}, \lambda_{2}}\left(u_{0} ; r\right) \backslash W_{\lambda_{1}, \lambda_{2}}\left(u_{0} ; \varepsilon\right)$.

It is assumed that

$$
\begin{gathered}
p_{k}\left(t, x_{1}, \ldots, x_{l}\right)=\int_{0}^{1} \frac{\partial f\left(t, u_{0}(t)+\xi x_{1}, \ldots, u_{0}^{(l-1)}(t)+\xi x_{l}\right)}{\partial x_{k}} d \xi \\
(k=1, \ldots, l), \\
\chi(s)=\left\{\begin{array}{l}
1 \quad \text { for } 0 \leq s \leq r, \\
2-\frac{s}{r} \quad \text { for } r<s<2 r, \\
0 \quad \text { for } s \geq 2 r,
\end{array}\right. \\
\widetilde{p}_{k}\left(t, x_{1}, \ldots, x_{l}\right)=p_{k}\left(t, x_{1} \chi\left(\sum_{j=1}^{l} \frac{\left|x_{j}\right|}{\sigma_{j, \lambda_{1}, \lambda_{2}(t)}}\right), \ldots, x_{l} \chi\left(\sum_{j=1}^{l} \frac{\left|x_{j}\right|}{\sigma_{j, \lambda_{1}, \lambda_{2}(t)}}\right)\right) \\
(k=1, \ldots, l), \\
\widetilde{\eta}_{i}\left(t, x_{1}, \ldots, x_{l}\right)=\eta_{i}\left(t, u_{0}(t)+x_{1} \chi\left(\sum_{j=1}^{l} \frac{\left|x_{j}\right|}{\sigma_{j, \lambda_{1}, \lambda_{2}}(t)}\right), \ldots, u_{0}^{(l-1)}(t)+\right. \\
\left.+x_{l} \chi\left(\sum_{j=1}^{l} \frac{\left|x_{j}\right|}{\sigma_{j, \lambda_{1}, \lambda_{2}}(t)}\right)\right) \quad(k=1, \ldots, l) .
\end{gathered}
$$

Let us consider the equation

$$
v^{(n)}=\sum_{k=1}^{l} \widetilde{p}_{k}\left(t, v, \ldots, v^{(l-1)}\right) v^{(k-1)}+\widetilde{\eta}_{i}\left(t, v, \ldots, v^{(l-1)}\right)
$$

for each $i$. By (1.37), (3.40)-(3.43) ascertain that the following inequalities are fulfilled on $] a, b\left[\times \mathbb{R}^{l}\right.$ :

$$
p_{1 k}(t) \leq \widetilde{p}_{k}\left(t, x_{1}, \ldots, x_{l}\right) \leq p_{2 k}(t) \quad(k=1, \ldots, l)
$$


and

$$
\left|\widetilde{\eta}_{i}\left(t, x_{1}, \ldots, x_{l}\right)-\eta_{0 i}(t)\right| \leq q_{i}(t)
$$

where

$$
q_{i}(t)= \begin{cases}\omega\left(t, r+\sum_{j=1}^{l} \frac{\left|u_{0}^{(j-1)}(t)\right|}{\sigma_{j, \lambda_{1}, \lambda_{2}}(t)}+\left|x_{k 0}\right|\right) & \text { for } \alpha \leq t \leq \beta, \\ \eta_{\lambda_{1}, \lambda_{2}}^{*}\left(t ; u_{0} ; r\right) & \text { for }] a, b[\backslash[\alpha, \beta] .\end{cases}
$$

From (3.37), (3.38) we readily obtain $\eta_{0 i}, q_{i} \in L_{n-1-\lambda_{1}, n-1-\lambda_{2}}(] a, b[; \mathbb{R})(i=$ $1,2, \ldots)$. However, since condition (1.8)is fulfilled, by virtue of Theorem 1.1 equation (3.44) has, for each natural number $i$, at least one solution satisfying $\left(1.2_{1}\right),\left(1.2_{2}\right)$.

Let $v_{0}$ be an arbitrary solution of problem $(3.44),\left(1.2_{1}\right),\left(1.2_{2}\right)$. Then either

or

$$
\text { vrai } \max \left\{\sum_{k=1}^{l} \frac{\left|v_{0}^{(k-1)}(t)\right|}{\sigma_{k, \lambda_{1}, \lambda_{2}}(t)}: a<t<b\right\}>r,
$$

$$
\text { vrai } \max \left\{\sum_{k=1}^{l} \frac{\left|v_{0}^{(k-1)}(t)\right|}{\sigma_{k, \lambda_{1}, \lambda_{2}}(t)}: a<t<b\right\} \leq r .
$$

If the latter inequality is fulfilled, then in view of (3.40)-(3.43) and the equality

$$
\begin{aligned}
f\left(t, u_{0}(t)+x_{1}, \ldots,\right. & \left.u_{0}^{(l-1)}(t)+x_{l}\right)-f\left(t, u_{0}(t), \ldots, u_{0}^{(l-1)}(t)\right)= \\
& =\sum_{k=1}^{l} p_{k}\left(t, x_{1}, \ldots, x_{l}\right) x_{k}
\end{aligned}
$$

it is obvious that the function $u(t)=u_{0}(t)+v_{0}(t)$ is a solution of problem $(3.39),\left(1.2_{1}\right),\left(1.2_{2}\right)$ contained in $W_{\lambda_{1}, \lambda_{2}}\left(u_{0} ; r\right)$. However, by our assumption, in the case under consideration problem (3.39),(1.2 $),\left(1.2_{2}\right)$ has at least one solution $\widetilde{u}$ such that $\widetilde{u} \in W_{\lambda_{1}, \lambda_{2}}\left(u_{0} ; r\right) \backslash W_{\lambda_{1}, \lambda_{2}}\left(u_{0} ; \varepsilon\right)$.

It is obvious that the function $v(t)=\widetilde{u}(t)-u_{0}(t)$ is a solution of problem $(3.44),\left(1.2_{1}\right),\left(1.2_{2}\right)$ satisfying the inequality

$$
\text { vrai } \max \left\{\sum_{k=1}^{l} \frac{\left|v^{(k-1)}(t)\right|}{\sigma_{k, \lambda_{1}, \lambda_{2}}(t)}: a<t<b\right\}>\varepsilon .
$$

Thus, for each natural number $i$ problem $(3.44),\left(1.2_{1}\right),\left(1.2_{2}\right)$ has the solution $v_{i}$ satisfying the condition

$$
\text { vrai } \max \left\{\sum_{k=1}^{l} \frac{\left|v_{i}^{(k-1)}(t)\right|}{\sigma_{k, \lambda_{1}, \lambda_{2}}(t)}: a<t<b\right\}>\varepsilon .
$$


Let $\rho_{0}$ be the number from Proposition 2.1. Then in view of (1.8) and (3.45) the inequality

$$
\begin{aligned}
& \left|v_{i}^{(k-1)}(t)\right| \leq \rho_{0}\left|\widetilde{\eta}_{i}\left(\cdot, v_{i}(\cdot), \ldots, v_{i}^{(l-1)}(\cdot)\right)\right|_{n-1-\lambda_{1}, n-1-\lambda_{2}} \sigma_{k, \lambda_{1}, \lambda_{2}}(t) \\
& \text { for } a<t<b \quad(k=1, \ldots, l)
\end{aligned}
$$

is fulfilled for each $i$.

By (3.37), (3.38) and (3.46) we have

$$
\begin{gathered}
\eta^{*}=\sup \left\{\left|\widetilde{\eta}\left(\cdot, v_{i}(\cdot), \ldots, v_{i}^{(l-1)}(\cdot)\right)\right|_{n-1-\lambda_{1}, n-1-\lambda_{2}}: i=1,2, \ldots\right\} \leq \\
\leq \sup \left\{\left|\eta_{0 i}(\cdot)\right|_{n-1-\lambda_{1}, n-1-\lambda_{2}}+\left|q_{i}(\cdot)\right|_{n-1-\lambda_{1}, n-1-\lambda_{2}}: i=1,2, \ldots\right\}<+\infty .
\end{gathered}
$$

Using (3.45), (3.46), (3.48) and the latter inequality, from (3.44) we have

$$
\begin{gathered}
\sum_{k=1}^{l}\left|v_{i}^{(k-1)}(t)\right| \leq \rho^{*}, \quad\left|v_{i}^{(n)}-\eta_{0 i}(t)\right| \leq p^{*}(t) \\
\text { for } \quad \alpha \leq t \leq \beta \quad(i=1,2, \ldots)
\end{gathered}
$$

where

$$
\begin{aligned}
\rho^{*} & =\eta^{*} \rho_{0} \max \left\{\sum_{k=1}^{l} \sigma_{k, \lambda_{1}, \lambda_{2}}(t): \alpha \leq t \leq \beta\right\}, \\
p^{*}(t) & =\eta^{*} \rho_{0} \sum_{k=1}^{l}\left(\left|p_{1 k}(t)\right|+\left|p_{2 k}(t)\right|\right) \sigma_{k, \lambda_{1}, \lambda_{2}}(t)+ \\
& +\omega\left(t, r+\sum_{j=1}^{l}\left(\frac{\left|u_{0}^{(j-1)}(t)\right|}{\sigma_{j, \lambda_{1}, \lambda_{2}}(t)}+\left|x_{j 0}\right|\right)\right)
\end{aligned}
$$

and $p^{*} \in L([\alpha, \beta] ; \mathbb{R})$. Moreover, since

$$
\max \left\{\left|\int_{s}^{t} \eta_{0 i}(\tau) d \tau\right|: \alpha \leq s \leq t \leq \beta\right\} \rightarrow 0 \text { for } i \rightarrow+\infty,
$$

it follows from (3.49) that the sequences $\left(v_{i}^{(k-1)}\right)_{i=1}^{+\infty}(k=1, \ldots, l)$ are uniformly bounded and equicontinuous on $[\alpha, \beta]$. Therefore, by Lemma $3.1[8]$, conditions (3.35), (3.36), (3.41), and (3.43) imply

$$
\begin{gathered}
\gamma_{i}=\max \left\{\left|\int_{\alpha}^{t} \widetilde{\eta}_{i}\left(\tau, v_{i}(\tau), \ldots, v_{i}^{(l-1)}(\tau)\right) d \tau\right|: \alpha \leq t \leq \beta\right\} \rightarrow 0 \\
\text { for } i \rightarrow+\infty
\end{gathered}
$$


In view of (3.37),(3.38),(3.46), and (3.50)

$$
\begin{gathered}
\left|\widetilde{\eta}_{i}\left(\cdot, v_{i}(\cdot), \ldots, v_{i}^{(l-1)}(\cdot)\right)\right|_{n-1-\lambda_{1}, n-1-\lambda_{2}} \leq \\
\leq\left(2 \gamma_{j}(b-a)^{2 n-2-\lambda_{1}-\lambda_{2}}+\frac{1}{i}\left[(b-a)^{n-1-\lambda_{1}}+(b-a)^{n-1-\lambda_{2}}\right]\right) \rightarrow 0 \\
\text { for } i \rightarrow+\infty
\end{gathered}
$$

and now from (3.48) we find

$$
\operatorname{vrai} \max \left\{\sum_{k=1}^{l} \frac{\left|v_{i}^{(k-1)}(t)\right|}{\sigma_{k, \lambda_{1}, \lambda_{2}}(t)}: a<t<b\right\} \rightarrow 0 \text { for } i \rightarrow+\infty .
$$

But this contradicts (3.47), which proves the theorem.

Theorem 1.6 immediately follows from Theorems 1.2 and 1.5.

\section{REFERENCES}

1. P.W. Bates and G.B. Gustafson, Green's function inequalities for twopoint boundary value problem. Pacif. J. Math. 59(1975), No. 2, 327-343.

2. Ch.-J. de la Vallée-Poussin, Sur l'equation differentielle lineaire du second ordre. Determination d'une integrale par deux valeurs assignees. Extension aux equation d'ordre n. J. Math. Pures et Appl. 8(1929), No. $2,125-144$.

3. E.S. Chichkin, Theorem on a differential inequality for the multipoint boundary value problems. (Russian) Izv. Vyssh. Uchebn. Zaved. Mat. 2(1962), 170-179.

4. P. Edwards, Functional analysis. Theory and applications. (Tranlated into Russian) Mir, Moscow, 1969; English original: Holt, Rinehart and Winston, New York, 1965.

5. I.T. Kiguradze, Some singular boundary value problems for ordinary differential equations.(Russian) Tbilisi University Press, Tbilisi, 1975.

6. - - On solvability of the boundary value problem of de la ValléePoussin. (Russian) Differentsial'nye Uravneniya 21(1985), No. 3, 391-398.

7. — - On boundary value problems for high-order ordinary differential equations with singularities. (Russian) Uspekhi Mat. Nauk 41(1986), No. 4, 166-167.

8. — - Boundary value problems for systems of ordinary differential equations. (Russian) Current problems in mathematics. Newest results, vol. 30 (Russian) 3-103; Itogi nauki i tekhniki, Akad. Nauk SSSR, Vsesoyuzn. Inst. Nauchn. i Tekhnich. Inform., Moscow, 1987. 
9. A.Yu. Levin, Nonoscillation of solutions of the equation $x^{(n)}+$ $p_{1}(t) x^{(n-1)}+\cdots+p_{n}(t) x=0$. (Russian) Uspekhi Mat. Nauk 24(1969), No. 2(146), 43-96.

10. G.D. Tskhovrebadze, On a singular boundary value problem of the de la Vallée-Poussin type. (Russian) Proc. I.N. Vekua Inst. Appl. Math. Tbilisi State Univ. 31(1988), 179-194.

11. - - On the correctness of a singular problem of the de la ValléePoussin type. (Russian) Bull. Acad. Sci. Georgian SSR 135(1989), No. 3, 489-492.

12. - - On a class $V\left(t_{1}, \ldots, t_{m} ; n_{1}, \ldots, n_{m} ; \lambda_{1}, \lambda_{2}\right)$. Preprint. A. Razmadze Math. Inst. Acad. Sci. Rep. of Georgia, Tbilisi, 1991.

13. - On the existence, uniqueness, and correctness of the solution of a Vallée-Poussin type linear boundary value problem.Preprint. A. Razmadze Math. Inst. Acad. Sci. Rep. of Georgia, Tbilisi, 1991.

14. —- On a multipoint boundary value problem for linear ordinary differential equations with singularities. Arch. Math. (to appear).

(Received 7.07.1993)

Author's address:

A.Razmadze Mathematical Institute

Georgian Academy of Sciences

1, Z.Rukhadze St., 380093 Tbilisi

Republic of Georgia 